\title{
Leptonic sum rules from flavour models with modular symmetries
}

\section{J. Gehrlein ${ }^{a}$ and M. Spinrath ${ }^{b}$}

${ }^{a}$ High Energy Theory Group, Physics Department, Brookhaven National Laboratory, Upton, NY 11973, U.S.A.

${ }^{b}$ Department of Physics, National Tsing Hua University, Hsinchu 30013, Taiwan

E-mail: jgehrlein@bnl.gov, spinrath@phys.nthu.edu.tw

ABSTRACT: Sum rules in the lepton sector provide an extremely valuable tool to classify flavour models in terms of relations between neutrino masses and mixing parameters testable in a plethora of experiments. In this manuscript we identify new leptonic sum rules arising in models with modular symmetries with residual symmetries. These models simultaneously present neutrino mass sum rules, involving masses and Majorana phases, and mixing sum rules, connecting the mixing angles and the Dirac CP-violating phase. The simultaneous appearance of both types of sum rules leads to some non-trivial interplay, for instance, the allowed absolute neutrino mass scale exhibits a dependence on the Dirac CP-violating phase. We derive analytical expressions for these novel sum rules and present their allowed parameter ranges as well as their predictions at upcoming neutrino experiments.

Keywords: Neutrino Physics, Beyond Standard Model, CP violation

ARXiv EPrINT: 2012.04131 


\section{Contents}

1 Introduction 1

2 Mass sum rules 3

2.1 Observables 3

2.1.1 Neutrino mass scale 3

2.1.2 Neutrinoless double beta decay 4

3 A detailed example 5

4 More models with sum rules: formulas 12

4.1 A model with two modular $S_{4}$ groups 12

$\begin{array}{ll}4.2 & \text { A model with a modular } S_{4} \text { symmetry } \\ \end{array}$

$\begin{array}{lll}4.3 & \text { A model with modular } A_{5} \text { symmetry } & 16\end{array}$

5 More models with sum rules: phenomenological results 17

6 Summary and conclusions $\quad 24$

\section{Introduction}

One of the big open questions of the Standard Model (SM) of particle physics is the origin of neutrino masses and mixings. The observation of neutrino oscillations demonstrated that neutrinos are massive. However, they are predicted to be massless in the original formulation of the SM. Since then neutrino oscillations have been well established and the three mixing angles in the PMNS matrix have been measured to a good accuracy (see, e.g., [1] for a global analysis of all oscillation data), while right now we only have hints of leptonic CP violation (CPV) [1] and a precise measurement of the Dirac CPV phase is up to near-future experiments. On the other hand so far we only have an upper bound on the absolute mass scale of neutrinos from cosmology and beta decay [2] which shows that neutrinos are several orders of magnitude lighter than the other SM fermions.

Large theoretical effort has been devoted to understand the origin of neutrino masses and mixings (see [3-10] for recent reviews). Particularly challenging from the theoretical point of view is to explain the observed pattern of neutrino mixing consisting of two large and one small mixing angle. Arguably one of the most natural explanations of this pattern is provided by models based on non-Abelian discrete flavour symmetries. Testing the predictions of these models is of utmost importance to understand the origin of neutrino masses and mixing. Most of the discrete symmetry models of neutrino mixing and, more generally, of lepton flavour, predict correlations between several observables, making them 
testable at current or upcoming experiments. Two different correlations between neutrino observables exist in models with discrete symmetries: neutrino mass sum rules (for early papers see [11-15], for reviews see [16-21]), which, in the case of three neutrino mixing, involve the three light neutrino masses and the two Majorana CPV phases [22] of the PMNS neutrino mixing matrix, and leptonic mixing sum rules which relate the leptonic mixing angles and the Dirac CPV phase, for some early papers with such an explicit connection, see, e.g., $[23,24]$ and for some more recent reviews and systematic studies see, e.g., [5, 6, 25-31]. Neutrino mass sum rules can be tested in experiments measuring the absolute neutrino mass scale or experiments that provide information on the sum of the neutrino masses. A number of studies have shown, in particular, that checking the validity of the leptonic mixing sum rules using the currently available and prospective data on the neutrino mixing angles and the Dirac CPV phase is an extremely powerful method of discriminating between different discrete symmetry models and, more generally, of testing the non-Abelian discrete symmetry approach to the neutrino mixing problem (see, e.g., [32-35]).

Recently, a generalisation of the discrete symmetry approach has been proposed in [36]. In this approach modular invariance plays the role of the flavour symmetry and couplings of the theory are modular forms of a certain level $N$. In the simplest realisation of this idea, the vacuum expectation value of a complex field (modulus) is the only source of flavour symmetry breaking, which leads to a reduction of free parameters in the respective models compared to the models based on the standard discrete symmetry approach where several copies of scalars (flavons) need to be introduced to break the flavour symmetry. This reduction of the parameters in models with modular symmetry leads to a new appealing feature, namely, the existence of new sum rules since the neutrino masses, neutrino mixing and the CPV phases are simultaneously determined by the modular symmetry typically in terms of a limited number of constant parameters. The simultaneous presence of neutrino mass and mixing sum rules makes these models highly predictive and can be used as a target for upcoming neutrino experiments.

In the following we will study models based on modular symmetries available in the literature which lead to new mass and mixing sum rules. We will focus on models which lead to the maximal number of sum rules, i.e., models with residual symmetries. In particular we will concentrate on sum rules which have been previously overlooked in these models. We will discuss their predictions for the bounds on the lightest neutrino mass, the observable in neutrinoless double beta decay, the kinematic neutrino mass as well as the mixing parameters. Our results can provide a link between model building, phenomenology, and experiments as they allow to study which models could be distinguished by the experiment and, in the case of an observation, the measurement can be directly linked to certain flavour models.

This manuscript is organized as follows: in section 2 we introduce the parametrization of mass sum rules and give general insights on the predictions of mass sum rules, in section 3 we provide an explicit, detailed example for sum rules in a model based on the modular symmetry $A_{4}$, section 4 is dedicated to a collection of models with residual symmetries present in the literature which feature sum rules whose predictions we show in section 5 and we summarize and conclude in section 6 . 


\section{Mass sum rules}

Before we look into actual models we want to discuss in some detail mass sum rules and how we can derive certain phenomenological predictions from them. For mixing sum rules, such a derivation is more straight-forward and we will not discuss any general statements about them here.

Mass sum rules relate the three light neutrino masses and two Majorana phases to each other. The existence of mass sum rules is not related to any symmetry nor related to a particular mass mechanism [37], it is merely the result of having less parameters than observables.

Using the complex mass eigenvalues $m_{i} \exp \left(-\mathrm{i} \phi_{i}\right)$ neutrino mass sum rules can be generally parametrised as

$$
\begin{aligned}
& s\left(m_{1}, m_{2}, m_{3}, \phi_{1}, \phi_{2}, \theta_{12}, \theta_{13}, \theta_{23}, \delta, d\right) \equiv \\
& f_{1}\left(\theta_{12}, \theta_{13}, \theta_{23}, \delta\right)\left(m_{1} \mathrm{e}^{-\mathrm{i} \phi_{1}}\right)^{d}+f_{2}\left(\theta_{12}, \theta_{13}, \theta_{23}, \delta\right)\left(m_{2} \mathrm{e}^{-\mathrm{i} \phi_{2}}\right)^{d}+m_{3}^{d} \stackrel{!}{=} 0,
\end{aligned}
$$

where $\phi_{1}, \phi_{2}$ are the Majorana phases and $f_{1}, f_{2}$ are model dependent, complex coefficients. Here we resemble for easier comparison the conventions used in [20,21]. For the original mass sum rules there was no explicit dependence of $f_{1}$ and $f_{2}$ on the mixing angles or the Dirac CPV phase. As we will see in the following this will not be the case anymore for sum rules in models with modular symmetries. Here the coefficients are functions of the mixing parameters leading to a strikingly different phenomenology of mass sum rules in models with and without modular symmetries. Starting from the parametrisation in eq. (2.1) we will discuss in the following how one can derive expressions for observables which are affected by the existence of a mass sum rule.

\subsection{Observables}

We want to discuss here some general formulas which will be useful to derive constraints on observables in models which have a mass sum rule. As mass sum rules involve the Majorana phases the ideal observable to test them is the observable in neutrinoless double beta decay $\left|m_{e e}\right|$. However, mass sum rules also provide a constraint on the absolute neutrino mass scale.

\subsubsection{Neutrino mass scale}

We begin with a formula for the lower and upper bound on neutrino masses assuming the presence of a mass sum rule. From experiments we have information on two neutrino mass squared differences hence it is possible to rewrite two of the masses as

$$
m_{2}=\sqrt{m_{1}^{2}+\Delta m_{21}^{2}}, m_{3}=\sqrt{m_{1}^{2}+\Delta m_{31}^{2}},
$$

which applies to both mass orderings. Normal mass ordering (NO) means: $m_{1}<m_{2}<m_{3}$ and inverted mass ordering (IO): $m_{3}<m_{1}<m_{2}$.

Looking at the mass sum rule from a geometrical point of view as a triangle in the complex plane, cf. $[16,17]$, it is clear that the most extremal masses can be achieved when 
the triangle degenerates into a line, i.e. when $f_{i}\left(m_{i} \mathrm{e}^{-\mathrm{i} \phi_{i}}\right)^{d}$ are real. In this case

$$
m_{3}^{d}=|| f_{1}\left|m_{1}^{d}+\right| f_{2}\left|m_{2}^{d}\right| \text { or } m_{3}^{d}=|| f_{1}\left|m_{1}^{d}-\right| f_{2}\left|m_{2}^{d}\right| .
$$

Replacing $m_{3}$ and $m_{2}$ with eq. (2.2) in this expression and solving for $m_{1}$ we obtain, for instance, for $d=1$,

$$
\begin{aligned}
m_{1}^{2}= & \frac{\Delta m_{21}^{2}\left|f_{2}\right|^{2}\left(\left|f_{1}\right|^{2}-\left|f_{2}\right|^{2}+1\right)+\Delta m_{31}^{2}\left(\left|f_{1}\right|^{2}+\left|f_{2}\right|^{2}-1\right)}{\left|f_{2}\right|^{4}-2\left(\left|f_{1}\right|^{2}+1\right)\left|f_{2}\right|^{2}+\left(\left|f_{1}\right|^{2}-1\right)^{2}} \\
& \pm \frac{2\left|f_{1}\right|\left|f_{2}\right| \sqrt{\Delta m_{31}^{2}\left(\Delta m_{21}^{2}\left(\left|f_{1}\right|^{2}-1\right)+\Delta m_{31}^{2}\right)+\Delta m_{21}^{2}\left|f_{2}\right|^{2}\left(\Delta m_{21}^{2}-\Delta m_{31}^{2}\right)}}{\left|f_{2}\right|^{4}-2\left(\left|f_{1}\right|^{2}+1\right)\left|f_{2}\right|^{2}+\left(\left|f_{1}\right|^{2}-1\right)^{2}}
\end{aligned}
$$

corresponding to the upper and lower bound for the mass scale. As we will see later we will have here only $d=-1$ and $d=+1$. However, the equivalent formula for $d=-1$ is extremely lengthy and not insightful. For this reason we will present in the next section another approach leading to compact but implicit expressions for all values of $d$.

The lower and upper bound on the lightest mass impacts the measurement on the sum of the neutrino masses, $\sum m_{i}$, where the strongest current upper limit on the sum of the neutrino masses as measured by Planck is $\sum m_{i}<0.12 \mathrm{eV}$ [38] as well as the kinematic neutrino mass

$$
m_{\beta}^{2}=\sum_{i}\left|U_{e i}\right|^{2} m_{i}^{2},
$$

which can be measured with beta decay experiments like KATRIN which provides a current limit of $m_{\beta}<1.1 \mathrm{eV}[39]$ and is expected to reach a sensitivity of $0.2 \mathrm{eV}$ in the future. Note that the kinematic neutrino mass also depends explicitly on the leptonic mixing angles such that this observable gets also affected by the presence of a mixing sum rule.

\subsubsection{Neutrinoless double beta decay}

If neutrinos are Majorana particles, neutrinoless double beta decay is possible. In the minimal scheme the decay rate is related to the parameter combination

$$
\left|m_{e e}\right|=\left|m_{1} U_{e 1}^{2}+m_{2} U_{e 2}^{2}+m_{3} U_{e 3}^{2}\right|=\left|m_{1} c_{12}^{2} c_{13}^{2} \mathrm{e}^{-\mathrm{i} \phi_{1}}+m_{2} s_{12}^{2} c_{13}^{2} \mathrm{e}^{-\mathrm{i} \phi_{2}}+m_{3} s_{13}^{2} \mathrm{e}^{-2 \mathrm{i} \delta}\right| .
$$

Without a mass sum rule, this observable is being extremized when the Majorana phases take the values $2 \delta$ or $2 \delta+\pi$. Then the factor $\exp (-2 \mathrm{i} \delta)$ is a global unphysical factor. In the presence of a mass sum rule this easy relation does not hold anymore since the Majorana phases are not independent parameters.

In fact, we can use the mass sum rule to get expressions for $\phi_{1}$ and $\phi_{2}$. We begin with $\phi_{2}$ and solve the mass sum rule for $m_{2} \mathrm{e}^{-\mathrm{i} \phi_{2}}$ such that

$$
\left|m_{e e}\right|=\left|m_{1} c_{12}^{2} c_{13}^{2} \mathrm{e}^{-\mathrm{i} \phi_{1}}+\frac{s_{12}^{2} c_{13}^{2}}{f_{2}^{1 / d}}\left(-m_{3}^{d}-f_{1}\left(m_{1} \mathrm{e}^{-\mathrm{i} \phi_{1}}\right)^{d}\right)^{1 / d}+m_{3} s_{13}^{2} \mathrm{e}^{-2 \mathrm{i} \delta}\right| .
$$

For instance, for $d=1$ this simplifies to

$$
\left|m_{e e}\right|=\left|m_{1}\left(c_{12}^{2} c_{13}^{2}-\frac{f_{1}}{f_{2}} s_{12}^{2} c_{13}^{2}\right) \mathrm{e}^{-\mathrm{i} \phi_{1}}+m_{3}\left(s_{13}^{2} \mathrm{e}^{-2 \mathrm{i} \delta}-\frac{s_{12}^{2} c_{13}^{2}}{f_{2}}\right)\right| .
$$


We see that $\left|m_{e e}\right|$ now only depends on $\phi_{1}$ since we eliminated $m_{2}$ and $\phi_{2}$.

To eliminate $\phi_{1}$ we solve the mass sum rule for $f_{2}\left(m_{2} \mathrm{e}^{-\mathrm{i} \phi_{2}}\right)^{d}$ and multiply it with its complex conjugate so that

$$
\begin{aligned}
\left|f_{2}\right|^{2} m_{2}^{2 d} & =\left|f_{1}\right|^{2} m_{1}^{2 d}+m_{3}^{2 d}+2\left|f_{1}\right| m_{1}^{d} m_{3}^{d} \cos \left(\arg \left(f_{1}\right)-d \phi_{1}\right) \\
\Leftrightarrow \cos \left(\arg \left(f_{1}\right)-d \phi_{1}\right) & =\frac{\left|f_{2}\right|^{2} m_{2}^{2 d}-\left|f_{1}\right|^{2} m_{1}^{2 d}-m_{3}^{2 d}}{2\left|f_{1}\right| m_{1}^{d} m_{3}^{d}} \\
\Leftrightarrow \phi_{1} & =\frac{1}{d} \arg \left(f_{1}\right) \pm \frac{1}{d} \arccos \left(\frac{\left|f_{2}\right|^{2} m_{2}^{2 d}-\left|f_{1}\right|^{2} m_{1}^{2 d}-m_{3}^{2 d}}{2\left|f_{1}\right| m_{1}^{d} m_{3}^{d}}\right),
\end{aligned}
$$

where we use the main branch of $\arccos (x) \in[0, \pi]$ and the \pm then covers the full range of $\phi_{1}$. This expression for $\phi_{1}$ can be plugged into eq. (2.7) eliminating all Majorana phases and reintroducing the dependence on $m_{2}$. That is advantageous since for the masses (and mixing angles) we have experimental information contrary to the Majorana phases and $f_{1}$, $f_{2}$ and $d$ are given by the mass sum rule. The two solutions of eq. (2.11) will enclose an allowed region in a plot $\left|m_{e e}\right|$ versus lightest neutrino mass as we will see later.

Furthermore, we can also determine the bounds on the mass scale from this equation setting $\cos \left(\arg \left(f_{1}\right)-d \phi_{1}\right)= \pm 1$ and solving for the lightest neutrino mass. This is in general a complicated formula, but no problem for common computer algebra systems, in particular, after setting all parameters apart from the mass scale to numerical values. For a given set of $f_{1}, f_{2}, d$ and mass squared differences it can also happen that there are no real solutions for eq. (2.11) or that they are outside of the experimentally allowed region excluding that particular parameter set for the flavour model at hand.

\section{A detailed example}

As an explicit, detailed example for the new class of leptonic sum rules we analyse the model from [40] based on a modular $A_{4}$ symmetry. Let us begin first with the parameter counting to identify how many sum rules we expect. For the charged lepton sector two cases are present in this model, which nevertheless both just have three parameters describing the charged lepton masses which can be made real by unphysical phase transformations. The difference between the two cases is a constant mixing matrix, to which we will come back later.

Here we have to emphasize that the value of the moduli in this model is fixed to certain values, which respect a residual symmetry. In the charged lepton sector that is $\left\langle\tau_{l}\right\rangle=\mathrm{i} \infty$ for case I and $\left\langle\tau_{l}\right\rangle=-1 / 2+\mathrm{i} \sqrt{3} / 2$ for case II. Both values leave a residual $Z_{3}$ symmetry intact in the charged lepton sector. In the neutrino sector the modulus is fixed to $\left\langle\tau_{\nu}\right\rangle=\mathrm{i}$ preserving a $Z_{2}$ symmetry. 
The light neutrino mass matrix in this model has the structure

$$
\begin{aligned}
m_{\nu} & =a_{1}\left(\begin{array}{ccc}
2 & -1 & -1 \\
-1 & 2 & -1 \\
-1 & -1 & 2
\end{array}\right)+a_{2}\left(\begin{array}{lll}
1 & 0 & 0 \\
0 & 0 & 1 \\
0 & 1 & 0
\end{array}\right)+a_{3}\left(\begin{array}{lll}
0 & 0 & 1 \\
0 & 1 & 0 \\
1 & 0 & 0
\end{array}\right) \\
& =\frac{c}{\sqrt{3}}\left(\begin{array}{lll}
1 & 0 & 2 \\
0 & 2 & 1 \\
2 & 1 & 0
\end{array}\right)+\frac{a c}{3}\left(\begin{array}{ccc}
5 & -1 & -1 \\
-1 & 2 & 2 \\
-1 & 2 & 2
\end{array}\right)+\frac{b c}{3}\left(\begin{array}{ccc}
1 & 1 & 1 \\
1 & -2 & 4 \\
1 & 4 & -2
\end{array}\right) .
\end{aligned}
$$

Here we have three complex parameters and we can absorb one unphysical phase (the other two phases are relative) describing three masses, three mixing angles and three CPV phases. Therefore we expect in total four predictions which in the standard parametrisation are relations between observables. Since we want to write down a neutrino mass sum rule, which gives two relations, we can find two more relations between the mixing angles and the Dirac CP phase.

Let us begin with case I in this model. The neutrino mass matrix can be diagonalised by

$$
m_{\nu}^{\text {diag }}=U_{13}(\theta, \phi)^{T} U_{\mathrm{TBM}}^{T} m_{\nu} U_{\mathrm{TBM}} U_{13}(\theta, \phi)
$$

where we use the following phase convention for the so-called tri-bimaximal (TBM) mixing matrix [41]

$$
U_{\mathrm{TBM}}=\left(\begin{array}{ccc}
2 / \sqrt{6} & 1 / \sqrt{3} & 0 \\
-1 / \sqrt{6} & 1 / \sqrt{3} & -1 / \sqrt{2} \\
-1 / \sqrt{6} & 1 / \sqrt{3} & 1 / \sqrt{2}
\end{array}\right) .
$$

The matrix $U_{13}$ is a unitary rotation matrix

$$
U_{13}(\theta, \phi)=\left(\begin{array}{ccc}
\cos \theta & 0 & \mathrm{e}^{-\mathrm{i} \phi} \sin \theta \\
0 & 1 & 0 \\
-\mathrm{e}^{\mathrm{i} \phi} \sin \theta & 0 & \cos \theta
\end{array}\right),
$$

depending on the angle $\theta$ and the phase $\phi$ and which accounts for non-zero $\theta_{13}$. Note that we use a different sign convention for $\phi$ compared to [40].

The fact that the neutrino mixing matrix has such a simple structure is a consequence of the residual symmetry. The columns of the TBM matrix are indeed eigenvectors of the preserved $Z_{2}$ generator

$$
S=\frac{1}{3}\left(\begin{array}{ccc}
-1 & 2 & 2 \\
2 & -1 & 2 \\
2 & 2 & -1
\end{array}\right),
$$

which is a symmetry of the mass matrix eq. (3.1), cf. [40]. We will see such patterns again in the other models we consider.

Then the three neutrino masses are (see [40])

$$
m_{1} \mathrm{e}^{-\mathrm{i} \varphi_{1}}=c\left(z-\frac{1}{\sin 2 \theta}\right) \mathrm{e}^{\mathrm{i} \phi},
$$




$$
\begin{aligned}
& m_{2} \mathrm{e}^{-\mathrm{i} \varphi_{2}}=c(\sqrt{3}+\mathrm{i} z \sin \phi-\cot 2 \theta \cos \phi), \\
& m_{3} \mathrm{e}^{-\mathrm{i} \varphi_{3}}=c\left(z+\frac{1}{\sin 2 \theta}\right) \mathrm{e}^{-\mathrm{i} \phi}
\end{aligned}
$$

where $z=a \mathrm{e}^{-\mathrm{i} \phi}-b \mathrm{e}^{\mathrm{i} \phi}$. Note that we use here $\varphi_{i}$ instead of $\phi_{i}$ since on the left-hand side we do not have yet fixed a convention for the Majorana phases.

The coefficients of the mass sum rule, $f_{1}$ and $f_{2}$, are functions of $\theta$ and $\phi$

$$
\begin{aligned}
f_{1} & =-\mathrm{e}^{-2 \mathrm{i} \phi}-\mathrm{i} \mathrm{e}^{-\mathrm{i} \phi} f_{2} \sin \phi, \\
& =-\mathrm{e}^{-2 \mathrm{i} \phi} \frac{\sqrt{3} \sin (2 \theta)-\cos \phi \cos (2 \theta)-\mathrm{i} \sin \phi}{\sqrt{3} \sin (2 \theta)-\cos \phi \cos (2 \theta)+\mathrm{i} \sin \phi} \\
f_{2} & =-\mathrm{e}^{-\mathrm{i} \phi} \frac{2}{\sqrt{3} \sin (2 \theta)-\cos \phi \cos (2 \theta)+\mathrm{i} \sin \phi} .
\end{aligned}
$$

It is obvious from these expressions that $\left|f_{1}\right|=1$ while $\left|f_{2}\right|$ is non-trivial. In [40] the authors did not calculate explicitly a mass sum rule. This result is hence new. We will derive five different mass sum rules in this paper from which four had not been derived explicitly in the original model.

From the mixing sum rules of the model [40] we additionally obtain the following predictions for the mixing parameters as a function of $\theta$ and $\phi$ (these relations were found before in $[8,42])$

$$
\begin{aligned}
\sin ^{2} \theta_{12}(\theta) & =\frac{1}{3-2 \sin ^{2} \theta}, \\
\sin ^{2} \theta_{13}(\theta) & =\frac{2}{3} \sin ^{2} \theta \\
\sin ^{2} \theta_{23}(\theta, \phi) & =\frac{1}{2}+\frac{\sin \theta_{13}(\theta)}{2} \frac{\sqrt{2-3 \sin ^{2} \theta_{13}(\theta)}}{1-\sin ^{2} \theta_{13}(\theta)} \cos \phi, \\
\delta(\theta, \phi) & =\arcsin \left(-\frac{\sin \phi}{\sin 2 \theta_{23}(\theta, \phi)}\right),
\end{aligned}
$$

where we did not write the dependence on $\theta$ and $\phi$ completely explicit in the last two equations for better readability. These four equations allow us to fix $\theta$ and $\phi$ and then give two additional relations (predictions). These formulas together with the above coefficients for a mass sum rule form what we will call sum rule 1 (SR 1) case I.

In order to obtain the experimentally allowed ranges for the model parameters $\theta$ and $\phi$ we confront eqs. (3.11)-(3.14) with global neutrino oscillation data where we use the one-dimensional $\chi^{2}$-profiles for these parameters provided by nu-fit v5.0 [1] to determine the total $\chi^{2}(\theta, \phi)$

$$
\begin{aligned}
\chi^{2}(\theta, \phi)= & \left(\frac{\sin ^{2} \theta_{12}(\theta, \phi)-\sin ^{2} \theta_{12}}{\sigma\left(\sin ^{2} \theta_{12}\right)}\right)^{2}+\left(\frac{\sin ^{2} \theta_{13}(\theta, \phi)-\sin ^{2} \theta_{13}}{\sigma\left(\sin ^{2} \theta_{13}\right)}\right)^{2} \\
& +\left(\frac{\sin ^{2} \theta_{23}(\theta, \phi)-\sin ^{2} \theta_{23}}{\sigma\left(\sin ^{2} \theta_{23}\right)}\right)^{2}+\left(\frac{\delta(\theta, \phi)-\delta}{\sigma(\delta)}\right)^{2}
\end{aligned}
$$


The minimal $\chi^{2}$ for case I in this model is $\chi_{\min }^{2}=8.6$ for NO, while the minimal $\chi^{2}$ for IO is 19.7, higher than for NO due to the current mild preference of the global fit for NO which leads to a minimal $\chi^{2}$ for IO of 10.8 in the absence of sum rules. The $\chi^{2}$ difference between the case with and without sum rules is similar in both cases, 8.6 and 8.9 respectively.

For case II the charged lepton sector is non-diagonal such that a contribution to the PMNS matrix from the mixing matrix which diagonalises the charged lepton mass matrix arises. The relation between the PMNS matrix in case I and case II is, cf. [40],

$$
U_{\text {PMNS }}^{\mathrm{II}}=\left(\begin{array}{ccc}
-1 & 0 & 0 \\
0 & \mathrm{e}^{\mathrm{i} \pi / 3} & 0 \\
0 & 0 & \mathrm{e}^{-\mathrm{i} \pi / 3}
\end{array}\right) U_{\text {PMNS }}^{\mathrm{I}}\left(\begin{array}{ccc}
\mathrm{e}^{-\mathrm{i}(\phi+\pi / 2)} & 0 & 0 \\
0 & 1 & 0 \\
0 & 0 & \mathrm{e}^{\mathrm{i}(\phi-\pi / 2)}
\end{array}\right) .
$$

The light neutrino masses of case II are related to case I via

$$
\begin{aligned}
& m_{1}^{(\mathrm{II})} \mathrm{e}^{-\mathrm{i} \phi_{1}^{(\mathrm{II})}}=m_{1}^{(\mathrm{I})} \mathrm{e}^{-\mathrm{i} \phi_{1}^{(\mathrm{I})}} \mathrm{e}^{4 \mathrm{i} \phi}, \\
& m_{2}^{(\mathrm{II})} \mathrm{e}^{-\mathrm{i} \phi_{2}^{(\mathrm{II})}}=m_{2}^{(\mathrm{I})} \mathrm{e}^{-\mathrm{i} \phi_{2}^{(\mathrm{I})}}\left(-\mathrm{e}^{-2 \mathrm{i} \phi}\right),
\end{aligned}
$$

while the mixing angles do not change and we can use the fit result for $\theta$ and $\phi$ in both cases. The coefficients in the mass sum rule change nevertheless

$$
\begin{aligned}
& f_{1}^{(\mathrm{II})}=f_{1}^{(\mathrm{I})} \mathrm{e}^{-4 \mathrm{i} \phi}, \\
& f_{2}^{(\mathrm{II})}=-f_{2}^{(\mathrm{I})} \mathrm{e}^{2 \mathrm{i} \phi} .
\end{aligned}
$$

These changed coefficients together with the mixing sum rules in eqs. (3.11)-(3.14) form SR 1 case II.

Before turning to the predictions of the mass sum rule we discuss the predictions resulting from the mixing sum rules, eqs. (3.11)-(3.14). One obvious immediate consequence is that $\sin ^{2} \theta_{13}$ and $\sin ^{2} \theta_{12}$ are strongly correlated, i.e.

$$
3 \sin ^{2} \theta_{12}=\frac{1}{1-\sin ^{2} \theta_{13}} .
$$

Since $\theta_{13}$ is the best-known mixing angle this predicts a value of $\theta_{12}$ around $35.7^{\circ}$ close to the experimental upper $3 \sigma$ bound on $\theta_{12}$. More interesting though is the correlation between the rather badly known $\theta_{23}$ and the CPV phase $\delta$ depicted in figure 1 . The blue area encloses the region where we vary $\theta$ and $\phi$ within its $3 \sigma$ ranges for NO and IO, respectively, the blue star denoting the best fit point in the model. The dashed rectangle encloses the $3 \sigma$ ranges and the black star stands for the best fit point of nu-fit 5.0. Compared to the unconstrained fit the allowed regions are much smaller. We also see that the best fit of the model prefers $\theta_{23}$ in the upper octant. For most of the possible values of $\delta$ two degenerate solutions for $\theta_{23}$ in the upper and lower octant are possible due to the fact that eq. (3.14) is symmetric around $\theta_{23}=45^{\circ}$ while maximal atmospheric mixing is only possible if $\delta=3 \pi / 2$ (which has also been noticed by the authors of [40]). This is obvious from eq. (3.14) which states that for maximal $\theta_{23}$ the Dirac CP phase $\delta=-\phi$, independent 

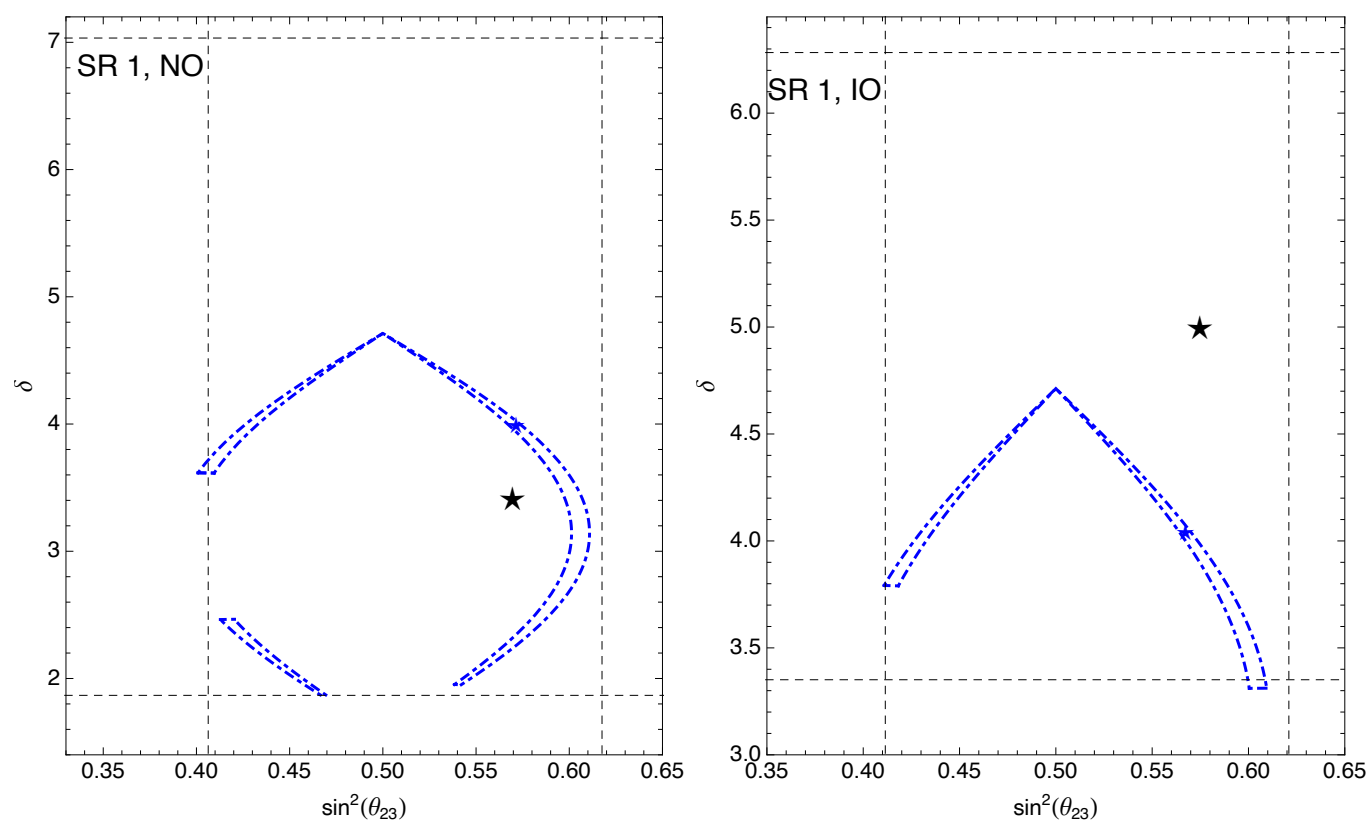

Figure 1. Correlations between $\delta$ and $\theta_{23}$ in blue for SR 1 for NO (left) and IO (right). The blue lines have been obtained by varying the model parameters $\theta$ and $\phi$ in their $3 \sigma$ ranges. The dashed lines show the experimental $3 \sigma$ ranges from nu-fit 5.0. The blue (black) star represents the best fit value with (without) the presence of SR 1.

of the value of $\theta$. Maximal $\theta_{23}$ can only be achieved if the second term in eq. (3.13) is zero, that is $\phi= \pm \pi / 2$ such that $\delta=\mp \pi / 2$. However, $\delta=\pi / 2$ is currently disfavoured by more than $3 \sigma$ by the global oscillation fit such that we only find the solution $3 \pi / 2$ in our model.

Turning now to the predictions of the mass sum rule. For the best fit values of $\theta$ and $\phi$ the coefficients of the mass sum rules are for NO in case I

$$
f_{1}^{(\mathrm{I})} \approx-0.11-0.99 \mathrm{i} \text { and } f_{2}^{(\mathrm{I})} \approx 2.02+1.80 \mathrm{i},
$$

and for IO

$$
f_{1}^{(\mathrm{I})} \approx-0.11-0.99 \mathrm{i} \text { and } f_{2}^{(\mathrm{I})} \approx 1.92+1.72 \mathrm{i}
$$

For case II we find

$$
f_{1}^{(\mathrm{II})} \approx 0.29+0.96 \mathrm{i} \text { and } f_{2}^{(\mathrm{II})} \approx 1.98-1.85 \mathrm{i},
$$

for $\mathrm{NO}$ and for $\mathrm{IO}$

$$
f_{1}^{(\mathrm{II})} \approx 0.99+0.09 \mathrm{i} \text { and } f_{2}^{(\mathrm{II})} \approx 2.58+0.11 \mathrm{i} .
$$

The results for $\left|m_{e e}\right|$ for NO and IO and case I and II are shown in figure 2. We see that SR 1 puts strong constraints on neutrinoless double beta decay, especially for IO where we also find that for the best fit values of $\theta$ and $\phi$ the SR cannot be fulfilled such that we only obtain a $3 \sigma$ range in IO. We have seen this constraining power already for the previous mass sum rules, cf. [18, 21, 43]. 

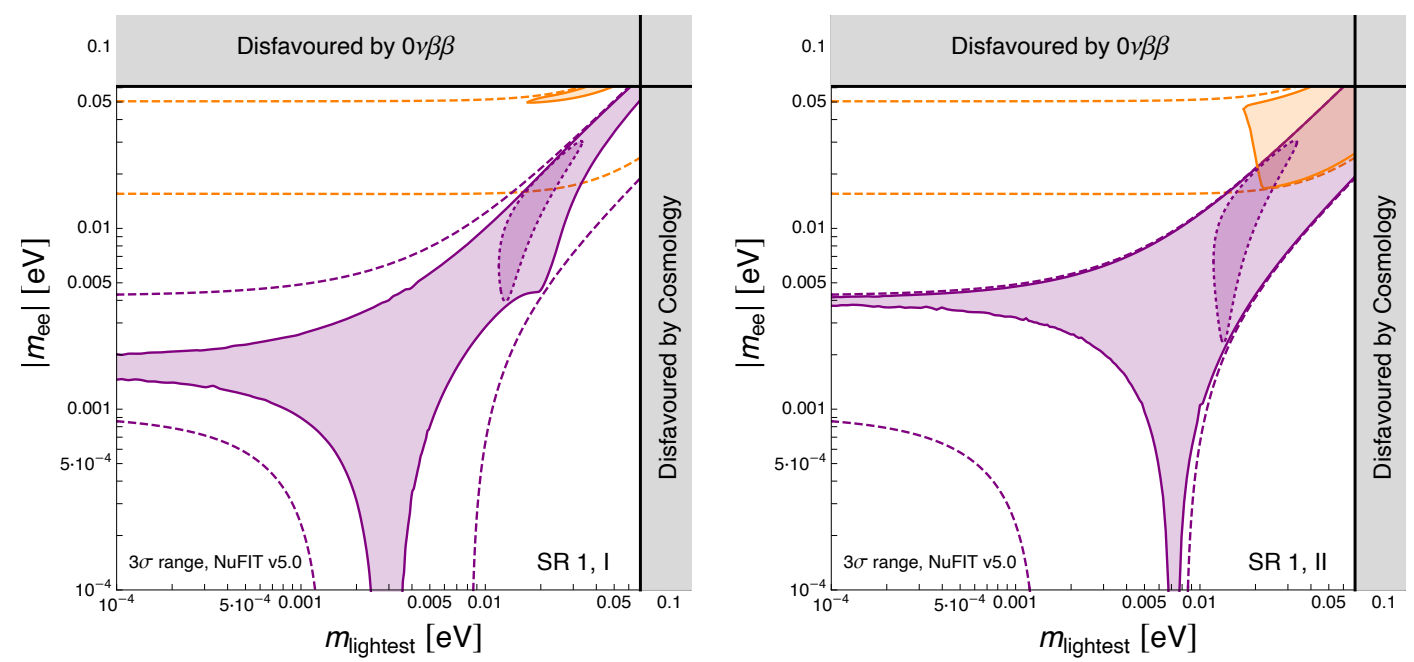

Figure 2. Allowed ranges for $\left|m_{e e}\right|$ for SR 1 in case I and case II. The purple/orange dashed region is the $3 \sigma$ allowed region without sum rules for NO/IO. The lightly (dark) shaded purple/orange regions is the $3 \sigma$ (best fit) allowed region for NO/IO. For more details, see main text.

There is nevertheless, one major difference in this model compared to the previous cases. For NO there is no lower bound on the mass scale which has been already noted in [40], which can be easily understood in our formalism. From the mass sum rule the absence of a lower bound, i.e., $m_{1}=0$, implies

$$
1 \ll \frac{m_{3}}{m_{2}}=\frac{\sqrt{\Delta m_{31}^{2}}}{\sqrt{\Delta m_{21}^{2}}}=\left|f_{2}\right|=\frac{2}{|\sqrt{3} \sin (2 \theta)-\cos \phi \cos (2 \theta)-\mathrm{i} \sin \phi|} .
$$

So if the denominator is close to zero this can be fulfilled which is indeed the case in our $3 \sigma$ region.

Similarly, there is also no upper bound on the mass scale for both NO and IO and we can understand this as well. An upper bound on the mass scale implies that there is a mass scale for which

$$
1<\left|\cos \left(\arg \left(f_{1}\right)-\phi_{1}\right)\right|=\left|\frac{\left|f_{2}\right|^{2} m_{2}^{2}-\left|f_{1}\right|^{2} m_{1}^{2}-m_{3}^{2}}{2\left|f_{1}\right| m_{1} m_{3}}\right|,
$$

where we have used that $d=1$. If there is no mass bound this implies that we can go to arbitrary large masses, where the mass splittings are negligible and still find a valid value for $\phi_{1}$. In that limit $m_{1} \approx m_{2} \approx m_{3}$ and

$$
\left|\cos \left(\arg \left(f_{1}\right)-\phi_{1}\right)\right| \approx\left|\frac{\left|f_{2}\right|^{2}-\left|f_{1}\right|^{2}-1}{2\left|f_{1}\right|}\right|=\left|1-\frac{\left|f_{2}\right|^{2}}{2}\right|,
$$

where we have used $\left|f_{1}\right|=1$. In the allowed $3 \sigma$ regions for both orderings $\left|f_{2}\right|$ can be smaller than two and hence there is no upper bound on the mass scale.

Finally, the true power of modular flavour models shows if we consider the correlations between mixing parameters and neutrino masses. In figure 3 we display the correlation 

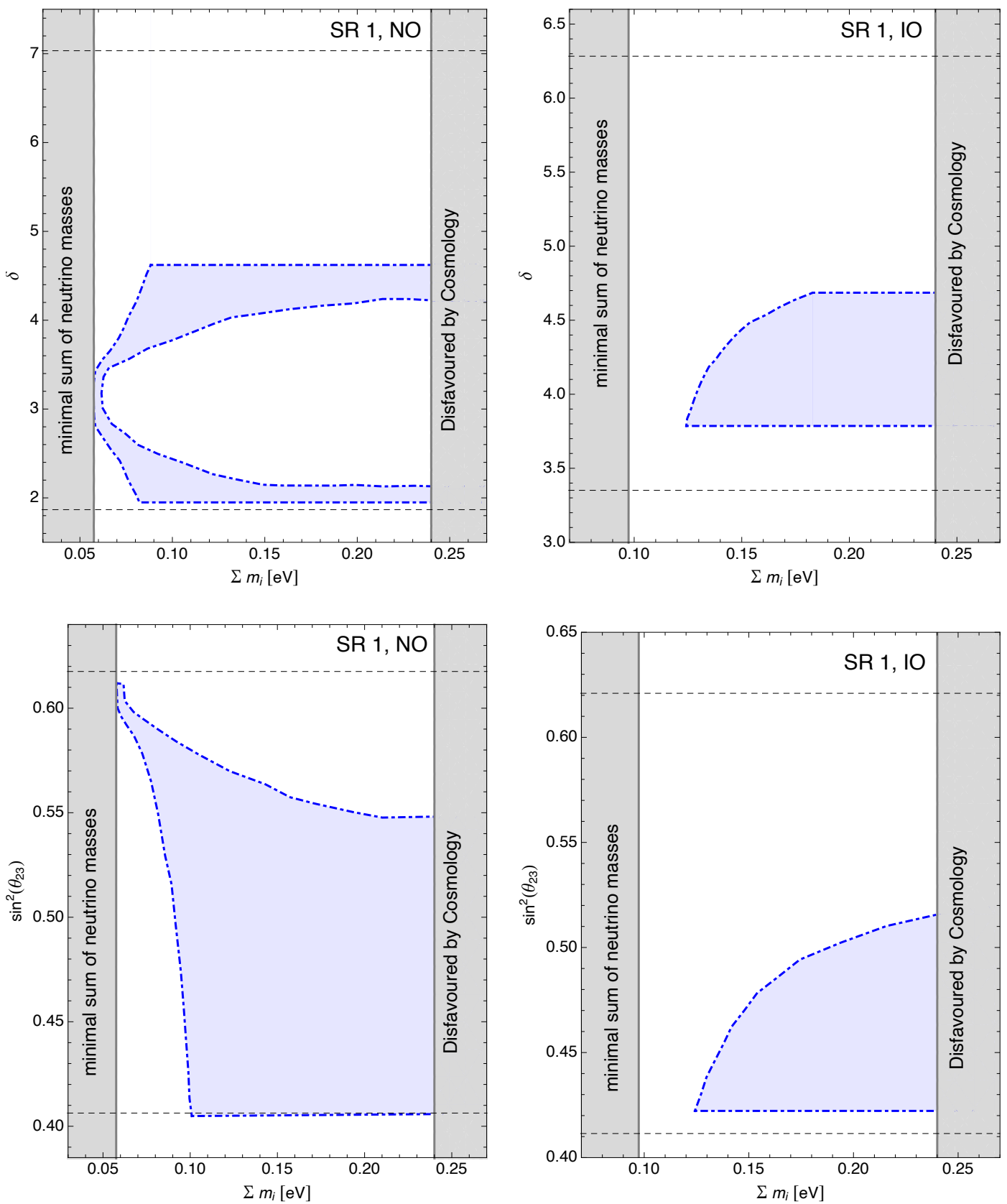

Figure 3. Correlation between $\delta$ (upper plots) or $\sin ^{2} \theta_{23}$ (lower plots) and the sum of the neutrino masses in SR 1 for NO (left plots) and IO (right plots) for $\theta, \phi$ and the mass splittings in their $3 \sigma$ ranges. The black dashed lines show the $3 \sigma$ allowed region without sum rules for NO/IO. The gray exclusion regions show the minimal value of the sum of the neutrino masses from oscillation experiments and the maximal value of the sum of the neutrino masses from cosmology. 
between $\theta_{23}$ and the sum of neutrino masses and $\delta$ and the sum of neutrino masses for NO and IO respectively. In particular, the minimal mass scale depends on the values of $\theta_{23}$ and $\delta$ which has also been seen in [40]. This model can therefore be constrained and potentially excluded by all(!) experiments measuring neutrino parameters like oscillation experiments but also experiments which are sensitive to the neutrino mass scale.

\section{More models with sum rules: formulas}

In the following we want to discuss more examples for flavour models based on modular symmetries which exhibit sum rules. There are many potential candidates but we will not consider models that have already been excluded or that have more parameters than observables in the neutrino sector. As the maximal number of sum rules is achieved when residual symmetries are present we will restrict ourselves to these cases. This means we want to restrict ourselves further on models where the values of the moduli are fixed on symmetry values. This allows to write down somewhat involved, but explicit expressions for mass sum rules as we have seen in section 3. To keep the number of parameters in the neutrino sector manageable we also do not consider radiative models here. In the general case, mass and/or mixing sum rules can still exist if there are less parameters than observables, but it can be difficult and not insightful to write them down. The existence of mass sum rules in particular is also suggested by the fact that many models with modular flavour symmetries have a lower bound on the neutrino mass scale which is a generic prediction of mass sum rules.

It is also interesting to note that in many cases the best fit value for the expectation value of the modulus, $\langle\tau\rangle$, is near a symmetry point, see, for instance, [44, 45], where formalisms have been developed which allows to expand around symmetry points.

This section provides the analytical expressions for the sum rules, in the next section we show a summary of the numerical results for them and compare them to our explicit example from the previous section. The models in this section have no particular order.

\subsection{A model with two modular $S_{4}$ groups}

In [46] a model with two modular $S_{4}$ groups was discussed. The modulus value $\left\langle\tau_{l}\right\rangle$ in the charged lepton sector is fixed to $\omega=\exp (2 \pi \mathrm{i} / 3)$ such that there is a residual $Z_{3}^{T}$ symmetry making the Yukawa matrix diagonal. In the neutrino sector $\left\langle\tau_{\nu}\right\rangle=(\mathrm{i}-1) / 2$ such that a $Z_{2}^{S U}$ symmetry is preserved with

$$
S U=\frac{1}{3}\left(\begin{array}{ccc}
-1 & 2 & 2 \\
2 & 2 & -1 \\
2 & -1 & 2
\end{array}\right)
$$

Due to this preserved generator which has the TBM matrix columns as eigenvector the neutrino mixing matrix has the trimaximal $\mathrm{TM}_{1}$ structure $[47,48]$

$$
U_{\mathrm{TM}_{1}}=U_{\mathrm{TBM}} U_{23}=U_{\mathrm{TBM}}\left(\begin{array}{ccc}
1 & 0 & 0 \\
0 & \cos \theta & \sin \theta \mathrm{e}^{-\mathrm{i} \phi} \\
0 & -\sin \theta \mathrm{e}^{\mathrm{i} \phi} & \cos \theta
\end{array}\right)
$$


with the same TBM matrix as in section 3 corrected by a 2-3 rotation. Note that we deviate here from the conventions of the 2-3 rotation in [46] for consistency with the rest of our paper.

The right-handed neutrino mass matrix in this model is

$$
M_{R}=a\left(\begin{array}{lll}
1 & 0 & 0 \\
0 & 0 & 1 \\
0 & 1 & 0
\end{array}\right)+b\left(\begin{array}{lll}
0 & 1 & 1 \\
1 & 1 & 0 \\
1 & 0 & 1
\end{array}\right)+c \sqrt{2}\left(\begin{array}{ccc}
2 & -1 & -1 \\
-1 & 2 & -1 \\
-1 & -1 & 2
\end{array}\right)-c \sqrt{3}\left(\begin{array}{ccc}
0 & 1 & -1 \\
1 & 2 & 0 \\
-1 & 0 & -2
\end{array}\right)
$$

and the Dirac neutrino mass matrix is

$$
M_{D}=y_{D} v_{u}\left(\begin{array}{lll}
1 & 0 & 0 \\
0 & 0 & 1 \\
0 & 1 & 0
\end{array}\right) \equiv y_{D} v_{u} P_{23}
$$

where we have defined the 2-3 permutation matrix $P_{23}$. We first diagonalise the righthanded neutrino mass matrix

$$
U_{\mathrm{TM}_{1}}^{T} M_{R} U_{\mathrm{TM}_{1}}=\operatorname{Diag}\left(\tilde{M}_{1}, \tilde{M}_{2}, \tilde{M}_{3}\right)
$$

where we eliminate $b$ using

$$
b=\frac{\mathrm{e}^{2 \mathrm{i} \phi}(a-3 \sqrt{2} c)+a+6 \sqrt{2} c \mathrm{e}^{\mathrm{i} \phi} \cot (2 \theta)}{-2+\mathrm{e}^{2 \mathrm{i} \phi}} .
$$

The parameters $\theta$ and $\phi$ can be determined from oscillation data as we will discuss later.

With the seesaw formula

$$
m_{\nu}=-M_{D}^{T} M_{R} M_{D}=-y_{D}^{2} v_{u}^{2} P_{23} U_{\mathrm{TM}_{1}} \operatorname{Diag}\left(\tilde{M}_{1}, \tilde{M}_{2}, \tilde{M}_{3}\right)^{-1} U_{\mathrm{TM}_{1}}^{T} P_{23}^{T}
$$

it is straight forward to write down the PMNS mixing matrix

$$
U_{\mathrm{PMNS}}=P_{23} U_{\mathrm{TBM}}^{*} U_{23}^{*} \Gamma_{i},
$$

where $\Gamma_{i}$ is a diagonal phase matrix which contains a global unphysical phase and the two physical Majorana phases. It is easy to derive that the light neutrino masses are given as

$$
\begin{aligned}
& -y_{D}^{2} v_{u}^{2} \tilde{m}_{1}^{-1}=\tilde{M}_{1}=c\left(-\frac{6 \sqrt{2} \mathrm{e}^{\mathrm{i} \phi} \cot (2 \theta)}{-2+\mathrm{e}^{2 \mathrm{i} \phi}}+\frac{6 \sqrt{2}}{-2+\mathrm{e}^{2 \mathrm{i} \phi}}+6 \sqrt{2}\right)-\frac{3 a}{-2+\mathrm{e}^{2 \mathrm{i} \phi}}, \\
& -y_{D}^{2} v_{u}^{2} \tilde{m}_{2}^{-1}=\tilde{M}_{2}=c \frac{\left(-3 \sqrt{2} \mathrm{e}^{\mathrm{i} \phi} \tan (\theta)\left(-2 \cot ^{2}(\theta)+\mathrm{e}^{2 \mathrm{i} \phi}\right)-6 \sqrt{2} \mathrm{e}^{2 \mathrm{i} \phi}\right)}{-2+\mathrm{e}^{2 \mathrm{i} \phi}}+\frac{3 a \mathrm{e}^{2 \mathrm{i} \phi}}{-2+\mathrm{e}^{2 \mathrm{i} \phi}}, \\
& -y_{D}^{2} v_{u}^{2} \tilde{m}_{3}^{-1}=\tilde{M}_{3}=c \frac{3\left(-2 \sqrt{2}+\sqrt{2} \mathrm{e}^{-\mathrm{i} \phi} \tan (\theta)\left(-2+\mathrm{e}^{2 \mathrm{i} \phi} \cot ^{2}(\theta)\right)\right)}{-2+\mathrm{e}^{2 \mathrm{i} \phi}}+\frac{3 a}{-2+\mathrm{e}^{2 \mathrm{i} \phi}} .
\end{aligned}
$$

From that we can determine the coefficients for the mass sum rule

$$
f_{1}=\frac{1}{\cos ^{2} \theta-\mathrm{e}^{\mathrm{i} \phi} \sin (2 \theta)},
$$




$$
\begin{aligned}
f_{2} & =-\frac{\tan \theta+2 \mathrm{e}^{\mathrm{i} \phi}}{2 \mathrm{e}^{3 \mathrm{i} \phi}-\mathrm{e}^{2 \mathrm{i} \phi} \cot (\theta)}, \\
d & =-1 .
\end{aligned}
$$

From these equations it is noteworthy that the right-handed neutrino masses fulfill a mass sum rule with the same $f_{1}$ and $f_{2}$ but $d=+1$. The authors of [46] also derive a mass sum rule which leads to the same predictions as our sum rule here. We only differ in conventions. The advantage of our approach is that we can immediately compare the result to other sum rules derived here or already present in the literature like in [11-21].

From the PMNS matrix we can also easily determine the relations between $\theta, \phi$ and the mixing angles in the standard convention

$$
\begin{aligned}
& \sin \theta_{13}=\left|\left(U_{\mathrm{PMNS}}\right)_{13}\right|=\frac{\sin \theta}{\sqrt{3}}, \\
& \tan \theta_{12}=\frac{\left|\left(U_{\mathrm{PMNS}}\right)_{12}\right|}{\left|\left(U_{\mathrm{PMNS}}\right)_{11}\right|}=\frac{\cos \theta}{\sqrt{2}}, \\
& \tan \theta_{23}=\frac{\left|\left(U_{\mathrm{PMNS}}\right)_{23}\right|}{\left|\left(U_{\mathrm{PMNS}}\right)_{33}\right|}=\left|\frac{\cos \theta+\sqrt{\frac{2}{3}} \mathrm{e}^{\mathrm{i} \phi} \sin \theta}{\cos \theta-\sqrt{\frac{2}{3}} \mathrm{e}^{\mathrm{i} \phi} \sin \theta}\right| .
\end{aligned}
$$

To derive an expression for the Dirac CP phase we first calculate

$$
\Delta=\frac{\left(U_{\mathrm{PMNS}}\right)_{11}^{*}\left(U_{\mathrm{PMNS}}\right)_{13}\left(U_{\mathrm{PMNS}}\right)_{31}\left(U_{\mathrm{PMNS}}\right)_{33}^{*}}{s_{12} s_{23} c_{12} c_{13}^{2} c_{23} s_{13}}+\frac{c_{12} c_{23} s_{13}}{s_{12} s_{23}}
$$

which is basis independent and in the standard parametrisation just $\exp (-\mathrm{i} \delta)$. Therefore

$$
\tan \delta=-\frac{\Im(\Delta)}{\Re(\Delta)}=-\frac{5+\cos (2 \theta)}{1+5 \cos (2 \theta)} \tan \phi .
$$

As we can see $\theta_{13}$ is determined by $\theta$ which implies $\theta \ll 1$. In this case $\delta \approx-\phi$ such that maximal $\theta_{23}$ is only possible for $\phi=-\delta= \pm \pi / 2$ for small $\theta$.

The sum rules derived from this model will be our sum rule 2 (SR 2).

\subsection{A model with a modular $S_{4}$ symmetry}

In [49] a model with a modular $S_{4}$ symmetry is presented, where the authors mostly discussed the general case without residual symmetries. But they also entertain the possibility to fix moduli to special values, namely $\left\langle\tau_{l}\right\rangle=\omega=\exp (2 \pi \mathrm{i} / 3)$ respecting a $Z_{3}^{S T}$ and $\left\langle\tau_{\nu}\right\rangle=\mathrm{i}$ respecting $Z_{2}^{S}$ in the neutrino sector. In these cases the charged lepton sector is diagonal. The neutrino sector has a type I seesaw with the neutrino Yukawa matrix

$$
Y_{\nu}=g P_{23},
$$

and the right-handed neutrino mass matrix can be written as

$$
M_{R}=\left(\begin{array}{ccc}
4 g_{1} & (3+\sqrt{6}) g_{2} \omega-\sqrt{6} g_{3}+g_{3} & (1+\sqrt{6}) g_{2}-(\sqrt{6}-3) g_{3} \omega^{2} \\
* & 4 g_{2} & 4 g_{1}+\sqrt{6} \omega\left(g_{2} \omega-g_{3}\right) \\
* & * & 4 g_{3}
\end{array}\right) .
$$


Since the matrix is symmetric we have labelled obvious elements with ' $*$ ' for the sake of brevity. These matrices have the residual $Z_{2}^{S}$ symmetry generated by

$$
S=\frac{1}{3}\left(\begin{array}{ccc}
-1 & 2 \omega^{2} & 2 \omega \\
2 \omega & 2 & -\omega^{2} \\
2 \omega^{2} & -\omega & 2
\end{array}\right),
$$

which is apart from phases the same as in section 4.1.

It is then easy to find that $M_{R}$ can be diagonalised by

$$
U_{23}^{T} U_{S}^{T} M_{R} U_{S} U_{23}=\operatorname{Diag}\left(\hat{M}_{1}, \hat{M}_{2}, \hat{M}_{3}\right),
$$

where

$$
U_{S}=\left(\begin{array}{ccc}
\sqrt{\frac{2}{3}} \zeta^{-1} & \frac{1}{\sqrt{3}} \omega & 0 \\
\frac{1}{\sqrt{6}} \omega^{2} & \frac{1}{\sqrt{3}} \omega^{2} & \frac{1}{\sqrt{2}} \\
\frac{1}{\sqrt{6}} & \frac{1}{\sqrt{3}} & \frac{1}{\sqrt{2}} \zeta^{-1}
\end{array}\right)
$$

is a unitary matrix with $\zeta=\exp (\mathrm{i} \pi / 3)=\sqrt{\omega}$ and $U_{23}$ is the 2-3 rotation introduced in section 4.1. Up to phases $U_{S}$ is the TBM mixing matrix.

Following an approach similar to [40], cf. section 3, we redefine the parameters to find

$$
\begin{aligned}
U_{23}^{T} U_{S}^{T} M_{R} U_{S} U_{23}= & c\left[\left(\begin{array}{ccc}
1+\mathrm{i} \sqrt{3} & 0 & 0 \\
0 & -\mathrm{e}^{\mathrm{i} \phi} \sin (2 \theta) & \cos (2 \theta) \\
0 & \cos (2 \theta) & \mathrm{e}^{-\mathrm{i} \phi} \sin (2 \theta)
\end{array}\right)\right. \\
& +a\left(\begin{array}{ccc}
-1+\mathrm{i} \sqrt{3} & 0 & 0 \\
0 & -2 \mathrm{e}^{2 \mathrm{i} \phi} \sin ^{2} \theta \mathrm{e}^{\mathrm{i} \phi} \sin (2 \theta) \\
0 & \mathrm{e}^{\mathrm{i} \phi} \sin (2 \theta) & -2 \cos ^{2} \theta
\end{array}\right) \\
& \left.+b\left(\begin{array}{ccc}
0 & 0 & 0 \\
0 & 2 \cos ^{2} \theta & \mathrm{e}^{-\mathrm{i} \phi} \sin (2 \theta) \\
0 \mathrm{e}^{-\mathrm{i} \phi} \sin (2 \theta) & 2 \mathrm{e}^{2 \mathrm{i} \phi} \sin ^{2} \theta
\end{array}\right)\right] .
\end{aligned}
$$

This matrix can be made diagonal by choosing $\theta$ and $\phi$ appropriately and we will use that

$$
b=-\mathrm{e}^{\mathrm{i} \phi} \cot (2 \theta)-a \mathrm{e}^{2 \mathrm{i} \phi} .
$$

The light neutrino mass matrix is given by the seesaw formula

$$
m_{\nu}=-v_{u}^{2} Y_{\nu}^{T} M_{R}^{-1} Y_{\nu}=-v_{u}^{2} g^{2} P_{23} U_{S} U_{23} \operatorname{Diag}\left(\tilde{M}_{1}, \tilde{M}_{2}, \tilde{M}_{3}\right)^{-1} U_{23}^{T} U_{S}^{T} P_{23}^{T} .
$$

The PMNS matrix is therefore given by

$$
U_{\mathrm{PMNS}}=P_{23} U_{S}^{*} U_{23}^{*} \Gamma_{i}
$$

where $\Gamma_{i}$ is again a diagonal phase matrix and $U_{\mathrm{PMNS}}^{T} m_{\nu} U_{\mathrm{PMNS}}=\operatorname{Diag}\left(m_{1}, m_{2}, m_{3}\right)$. Again the complex masses from the light and heavy neutrinos are related to each other

$$
-g^{2} v_{u}^{2} \tilde{m}_{1}^{-1}=\tilde{M}_{1}=c(a(-1+\mathrm{i} \sqrt{3})+1+\mathrm{i} \sqrt{3}),
$$




$$
\begin{aligned}
& -g^{2} v_{u}^{2} \tilde{m}_{2}^{-1}=\tilde{M}_{2}=c\left(-2 a \mathrm{e}^{2 \mathrm{i} \phi}-\mathrm{e}^{\mathrm{i} \phi} \cot \theta\right), \\
& -g^{2} v_{u}^{2} \tilde{m}_{3}^{-1}=\tilde{M}_{3}=c\left(-2 a+\mathrm{e}^{-\mathrm{i} \phi} \tan \theta\right),
\end{aligned}
$$

from which we can derive the mass sum rule coefficients

$$
\begin{aligned}
f_{1} & =\frac{2 /(\cos \theta \sin \theta)}{(-2-2 \mathrm{i} \sqrt{3}) \mathrm{e}^{\mathrm{i} \phi}+\mathrm{i}(\mathrm{i}+\sqrt{3}) \cot \theta}=\frac{1}{\omega^{2} \mathrm{e}^{\mathrm{i} \phi} \sin (2 \theta)+\omega \cos ^{2} \theta}, \\
f_{2} & =-\frac{\left(\mathrm{i}+\sqrt{3}+2(-\mathrm{i}+\sqrt{3}) \mathrm{e}^{\mathrm{i} \phi} \cot \theta\right) \tan \theta}{2(-\mathrm{i}+\sqrt{3}) \mathrm{e}^{3 \mathrm{i} \phi}-(\mathrm{i}+\sqrt{3}) \mathrm{e}^{2 \mathrm{i} \phi} \cot \theta}=-\frac{(1+\omega) \tan \theta+2 \mathrm{e}^{\mathrm{i} \phi}}{2 \mathrm{e}^{3 \mathrm{i} \phi}-(1+\omega) \mathrm{e}^{2 \mathrm{i} \phi} \cot \theta}, \\
d & =-1 .
\end{aligned}
$$

The relations for the mixing angles and the Dirac CP phase are straight-forward to derive as in section 4.1

$$
\begin{aligned}
\sin \theta_{13} & =\frac{1}{\sqrt{3}} \sin \theta \\
\tan \theta_{12} & =\frac{1}{\sqrt{2}} \cos \theta \\
\tan \theta_{23} & =\left|\frac{2 \mathrm{e}^{\mathrm{i} \phi} \tan \theta+\sqrt{3 / 2}(1+\mathrm{i} \sqrt{3})}{3 \sqrt{2 / 3}-(1-\sqrt{3} \mathrm{i}) \mathrm{e}^{\mathrm{i} \phi} \tan \theta}\right|, \\
\tan \delta & =-\frac{(\cos (2 \theta)+5)(\sqrt{3} \sin \phi-3 \cos \phi)}{(5 \cos (2 \theta)+1)(\sqrt{3} \cos \phi+3 \sin \phi)} .
\end{aligned}
$$

To obtain maximal $\theta_{23}, \phi=5 \pi / 6$. Again since $\theta$ is small and with $\phi=5 \pi / 6$ the $\mathrm{CPV}$ phase is given as $\delta \approx \pm \pi / 2$ for maximal $\theta_{23}$.

The sum rules derived from this model will be our sum rule 3 (SR 3).

\subsection{A model with modular $A_{5}$ symmetry}

In [50] two $A_{5}$ models were studied, from which one model is already excluded. The authors only discuss Weinberg operators and the charged lepton sector is diagonal. For the remaining model they set $\left\langle\tau_{\nu}\right\rangle=\mathrm{i}$ such that a $Z_{2}^{S}$ symmetry is preserved. The generator $S$ can be made diagonal using golden ratio mixing [51]

$$
U_{\mathrm{GR}}=\left(\begin{array}{ccc}
\sqrt{\frac{\phi_{g}}{\sqrt{5}}} & \sqrt{\frac{1}{\phi_{g} \sqrt{5}}} & 0 \\
-\sqrt{\frac{1}{2 \phi_{g} \sqrt{5}}} & \sqrt{\frac{\phi_{g}}{2 \sqrt{5}}} & \frac{1}{\sqrt{2}} \\
-\sqrt{\frac{1}{2 \phi_{g} \sqrt{5}}} & \sqrt{\frac{\phi_{g}}{2 \sqrt{5}}} & -\frac{1}{\sqrt{2}}
\end{array}\right),
$$

where $\phi_{g}=(1+\sqrt{5}) / 2$ which also puts the neutrino matrix into block-diagonal form

$$
U_{\mathrm{GR}}^{T} m_{\nu} U_{\mathrm{GR}}=c\left(\begin{array}{lcc}
b & 0 & 1 \\
0 \frac{1}{2 \sqrt{5}}((1+\sqrt{5}) a-(1-\sqrt{5}) b-8) & 0 \\
1 & 0 & -a
\end{array}\right),
$$


after a reparametrisation. We can now apply a 1-3 rotation as in eq. (3.2) and with $b=-\mathrm{e}^{\mathrm{i} \phi} \cot (2 \theta)-a \mathrm{e}^{2 \mathrm{i} \phi}$ we find the complex neutrino masses

$$
\begin{aligned}
& \tilde{m}_{1}=c\left(-\mathrm{e}^{\mathrm{i} \phi} \cot (\theta)-a \mathrm{e}^{2 \mathrm{i} \phi}\right), \\
& \tilde{m}_{2}=\frac{c}{10}\left(\left(2(\sqrt{5}-5) \mathrm{e}^{\mathrm{i} \phi} \cot (2 \theta)-8 \sqrt{5}\right)+a\left((\sqrt{5}-5) \mathrm{e}^{2 \mathrm{i} \phi}+5+\sqrt{5}\right)\right), \\
& \tilde{m}_{3}=c\left(\mathrm{e}^{-\mathrm{i} \phi} \tan (\theta)-a\right),
\end{aligned}
$$

leading to the mass sum rule coefficients

$$
\begin{aligned}
f_{1} & =\mathrm{e}^{-2 \mathrm{i} \phi} \frac{(1-\sqrt{5}) \mathrm{e}^{2 \mathrm{i} \phi} \cot \theta+(\sqrt{5}+1) \tan \theta-8 \mathrm{e}^{\mathrm{i} \phi}}{(1-\sqrt{5}) \mathrm{e}^{2 \mathrm{i} \phi} \tan \theta+(\sqrt{5}+1) \cot \theta+8 \mathrm{e}^{\mathrm{i} \phi}}, \\
f_{2} & =\frac{10}{(\sqrt{5}-5) \mathrm{e}^{2 \mathrm{i} \phi} \sin ^{2} \theta+4 \sqrt{5} \mathrm{e}^{\mathrm{i} \phi} \sin (2 \theta)+(5+\sqrt{5}) \cos ^{2} \theta}, \\
d & =+1
\end{aligned}
$$

For the mixing sum rules in this case we find

$$
\begin{aligned}
\sin \theta_{13}= & \sqrt{\frac{1}{10}(5+\sqrt{5})} \sin \theta \\
\tan \theta_{12}= & \frac{2}{1+\sqrt{5}} \frac{1}{\cos \theta}, \\
\tan \theta_{23}= & \left|\frac{\sqrt{\sqrt{5} \phi_{g}}-\mathrm{e}^{-\mathrm{i} \phi} \tan \theta}{\sqrt{\sqrt{5} \phi_{g}}+\mathrm{e}^{-\mathrm{i} \phi} \tan \theta}\right|, \\
\tan \delta= & \frac{4 \sqrt{5+\sqrt{5}} \sin (\phi)\left(2(\sqrt{5}+2) \cos ^{2}(\theta)+1+\sqrt{5}\right)}{D_{\delta}}, \\
D_{\delta}= & 4 \sqrt{5+\sqrt{5}} \cos (\phi) \cos (2 \theta)((\sqrt{5}+2) \cos (2 \theta)+3+2 \sqrt{5}) \\
& +\sqrt{2} \sin (2 \theta)((5 \sqrt{5}+11) \cos (2 \theta)+19+9 \sqrt{5}) \cos \left(2 \theta_{23}\right) .
\end{aligned}
$$

Similar as for SR 1 we find that for maximal $\theta_{23}$ the phase $\phi= \pm \pi / 2$ and then $\delta=\phi$ independent from the value of $\theta$.

The sum rules derived from this model will be our sum rule 4 (SR 4).

\section{More models with sum rules: phenomenological results}

In this section we present an overview of our phenomenological results. Having provided the analytical expressions for the mass and mixing sum rules in the previous section we evaluate them now numerically to show their predictions for upcoming experiments and compare them to each other.

We begin with table 1 providing a summary of parameters of all mass sum rules studied in this manuscript including the symmetry groups the models are based on. The quoted numbers for the coefficients $f_{i}$ are determined for the best fit parameters of the model 


\begin{tabular}{|lccccccc|}
\hline SR & $d$ & $\left|f_{1}\right|$ & $\arg \left(f_{1}\right)$ & $\left|f_{2}\right|$ & $\arg \left(f_{2}\right)$ & Group & Ref. \\
\hline 1, I (NO) & 1 & 1.00 & -1.68 & 2.71 & 0.73 & $A_{4}$ & {$[40]$} \\
1, I (IO) & 1 & 1.00 & -1.68 & 2.58 & 0.73 & $A_{4}$ & {$[40]$} \\
1, II (NO) & 1 & 1.00 & 1.28 & 2.71 & -0.75 & $A_{4}$ & {$[40]$} \\
1, II (IO) & 1 & 1.05 & 0.09 & 2.58 & -0.64 & $A_{4}$ & {$[40]$} \\
\hline $2(\mathrm{NO})$ & -1 & 0.87 & 0.43 & 0.41 & -1.52 & $S_{4} \times S_{4}$ & {$[46]$} \\
$2(\mathrm{IO})$ & -1 & 1.13 & 0.56 & 0.57 & -0.79 & $S_{4} \times S_{4}$ & {$[46]$} \\
\hline $3(\mathrm{NO})$ & -1 & 0.87 & -1.66 & 0.42 & 2.69 & $S_{4}$ & {$[49]$} \\
$3(\mathrm{IO})$ & -1 & 1.13 & -1.54 & 0.60 & -2.86 & $S_{4}$ & {$[49]$} \\
\hline $4(\mathrm{NO})$ & 1 & 0.45 & -1.29 & 1.94 & 0.43 & $A_{5}$ & {$[50]$} \\
$4(\mathrm{IO})$ & 1 & 0.56 & -2.27 & 1.13 & 0.31 & $A_{5}$ & {$[50]$} \\
\hline
\end{tabular}

Table 1. Overview of parameters entering the mass sum rule for the models we considered here. The values for $\left|f_{i}\right|$ and $\arg \left(f_{i}\right)$ are calculated with the best fit values of the underlying model parameters. However, it should be noted that for SR 1 cases I and II the mass sum rule cannot be fulfilled for the $\theta, \phi$ at their best fit values in IO.

\begin{tabular}{|lccccc|}
\hline No. & $\theta_{\mathrm{bf}}\left[^{\circ}\right]$ & $\phi_{\mathrm{bf}}\left[^{\circ}\right]$ & $\theta_{3 \sigma}\left[^{\circ}\right]$ & $\phi_{3 \sigma}\left[^{\circ}\right]$ & $\chi_{\min }^{2}$ \\
\hline $1(\mathrm{NO})$ & 10.5 & 47.58 & {$[10.0-11.0]$} & {$[152.5-293.5] \oplus[219.7-250.7]$} & 8.6 \\
$1(\mathrm{IO})$ & 10.6 & 50.8 & {$[10.1-11.0]$} & {$[9.5-143.5]$} & 19.7 \\
\hline $2(\mathrm{NO})$ & 15.0 & 103.2 & {$[14.3-15.7]$} & {$[55.9-117.3] \oplus[251.1-262.3]$} & 3.3 \\
& & & & $\oplus[285.9-292.6]$ & \\
$2(\mathrm{IO})$ & 15.0 & 69.1 & {$[14.0-15.9]$} & {$[50.5-122.2]$} & 12.1 \\
\hline $3(\mathrm{NO})$ & 14.9 & 163.2 & {$[14.3-15.7]$} & {$[115.9-177.3] \oplus[311.1-322.3]$} & 3.3 \\
& & & & $\oplus[345.9-353.6]$ & \\
$3(\mathrm{IO})$ & 15.0 & 129.0 & {$[14.4-15.7]$} & {$[114.8-173.8]$} & 12.1 \\
\hline $4(\mathrm{NO})$ & 10.1 & 221.6 & {$[9.7-10.6]$} & {$[115.1-333.4]$} & 3.8 \\
$4(\mathrm{IO})$ & 10.1 & 296.1 & {$[9.7-10.6]$} & {$[185.7-336.1]$} & 16.5 \\
\hline
\end{tabular}

Table 2. Overview of the ranges of the model parameters for all sum rules. For some sum rules there are multiple disjoint $3 \sigma$ regions for $\phi$. We also include the minimal value of the $\chi^{2}$ in these models. In IO the minimal $\chi^{2}$ without sum rules is 10.8 whereas it is zero in NO.

which we collected in table 2 together with their $3 \sigma$ ranges. We observe that for the best fit values of the model parameters the coefficients of the sum rules are $\left|f_{i}\right| \sim \mathcal{O}(1)$ however as we have seen in section 3 in the $3 \sigma$ range of $\theta$ and $\phi$ the coefficients can actually have a considerable range. Furthermore, it is notable that we only found sum rules with $d= \pm 1$ different from the conventional mass sum rules, where other values are possible as well, cf. $[16,18,20,21,37]$.

Figure 4 shows the allowed ranges for $\left|m_{e e}\right|$ over the lightest neutrino mass, $m_{\text {lightest }}$, for all sum rules considered in this manuscript. Additionally, we show in table 3 the lower bounds for the lightest neutrino mass, the sum of the neutrino masses, $\left|m_{e e}\right|$, and $m_{\beta}$ in the models. A general prediction of mass sum rules in models with modular symmetries are rather large values for the lightest neutrino mass $m_{\text {lightest }}>0.01 \mathrm{eV}$, which has also 

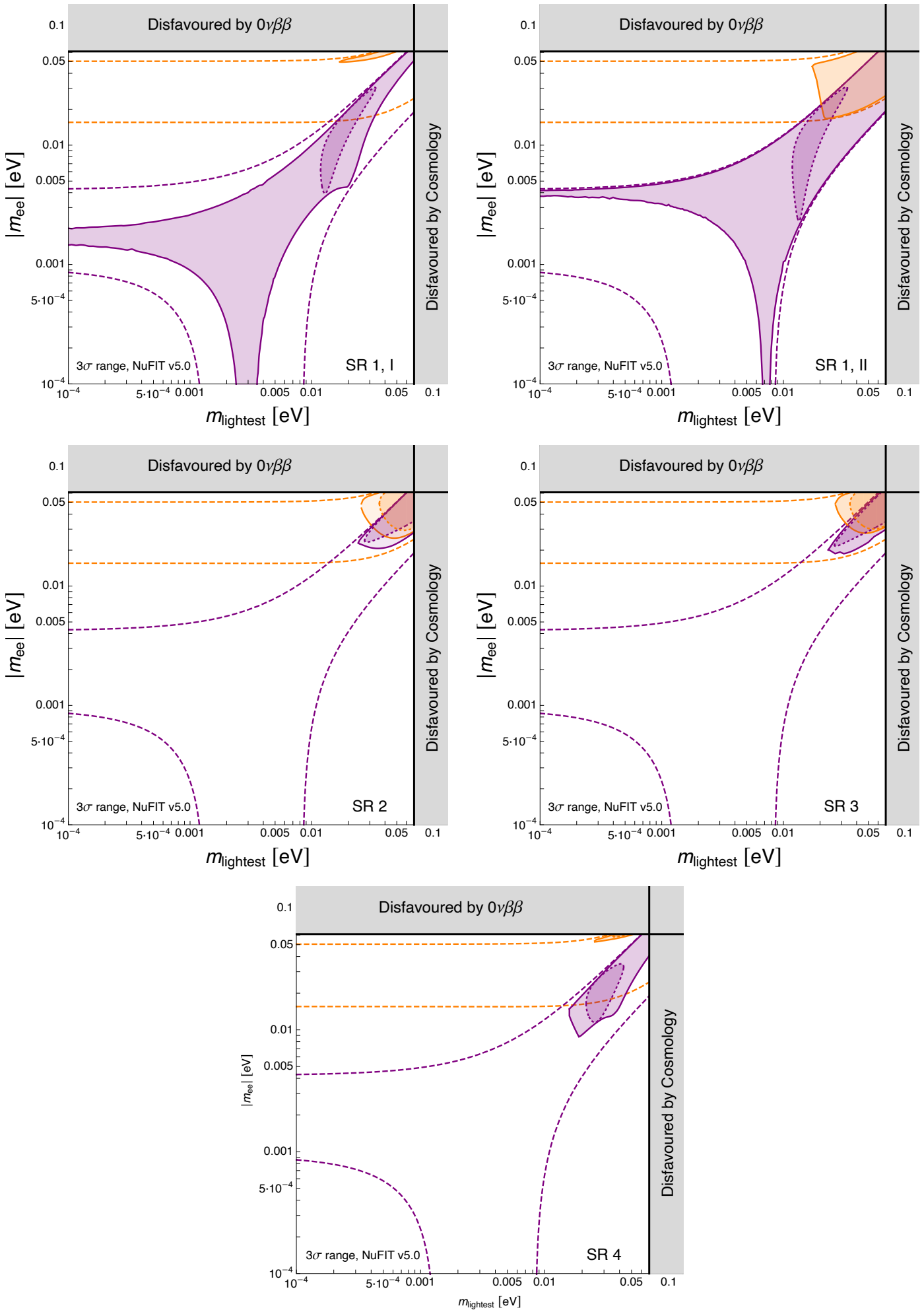

Figure 4. Allowed ranges for $\left|m_{e e}\right|$ for all sum rules. The purple/orange dashed region is the $3 \sigma$ allowed region without sum rules for $\mathrm{NO} / \mathrm{IO}$. The lightly (dark) shaded purple/orange region is the $3 \sigma$ (best fit) allowed region for $\mathrm{NO} / \mathrm{IO}$. For more details, see main text. 


\begin{tabular}{|llllll|}
\hline No. & $m_{\text {lightest }}$ & $\sum m_{i}$ & $\left|m_{e e}\right|$ & $m_{\beta}$ & Ref. \\
\hline 1, I (NO) & $\geq 0$ & $\geq 57.6$ & $\geq 0$ & $\geq 8.5$ & {$[40]$} \\
1, I $(\mathrm{IO})$ & $\geq 17.3$ & $\geq 124.2$ & $\geq 49.6$ & $\geq 52.9$ & {$[40]$} \\
1, II $(\mathrm{NO})$ & $\geq 0$ & $\geq 57.6$ & $\geq 0$ & $\geq 8.5$ & {$[40]$} \\
1, II $(\mathrm{IO})$ & $\geq 17.3$ & $\geq 124.2$ & $\geq 16.7$ & $\geq 52.9$ & {$[40]$} \\
\hline $2(\mathrm{NO})$ & $\geq 24.1$ & $\geq 104.5$ & $\geq 20.7$ & $\geq 25.5$ & {$[46]$} \\
$2(\mathrm{IO})$ & $\geq 25.4$ & $\geq 138.6$ & $\geq 25.2$ & $\geq 56.1$ & {$[46]$} \\
\hline $3(\mathrm{NO})$ & $\geq 23.4$ & $\geq 102.8$ & $\geq 18.7$ & $\geq 24.9$ & {$[49]$} \\
$3(\mathrm{IO})$ & $\geq 26.8$ & $\geq 141.3$ & $\geq 26.8$ & $\geq 56.7$ & {$[49]$} \\
\hline $4(\mathrm{NO})$ & $\geq 15.3$ & $\geq 84.3$ & $\geq 8.8$ & $\geq 17.4$ & {$[50]$} \\
$4(\mathrm{IO})$ & $\geq 24.8$ & $\geq 137.5$ & $\geq 52.7$ & $\geq 55.7$ & {$[50]$} \\
\hline
\end{tabular}

Table 3. Overview of lower bounds on various observables derived from the new mass sum rules discussed in this paper. Masses are given in meV. For more details, see main text.

been noticed in [36]. Our results do not only support this observation but they also provide a rationale for this. Namely, the existence of mass sum rules which usually can only be fulfilled for rather large mass scales as for $\mathcal{O}(1)$ coefficients the neutrino masses need to be of similar order to fulfill the sum rule. This statement however changes for certain parameter regions where the coefficients can be drastically different from $\mathcal{O}(1)$ as we have seen for SR 1 in section 3.

Furthermore, SR 2 and 3 do not predict an upper limit on the mass scale in the best fit region for $\theta$ and $\phi$ unlike SR 1 and SR 4 (see figures 2 and 4). Related to the preference for large neutrino mass scales only SR 1 cases I and II predict values of $\left|m_{e e}\right|$ below $10^{-3} \mathrm{eV}$ which are generically difficult to probe experimentally. The predicted parameter space for SR 2, 3, 4 and parts of the parameter space for SR 1 lie within the testable range of future experiments for neutrinoless double beta decay which will probe $\left|m_{e e}\right| \gtrsim(10-50) \mathrm{meV}$ [52] as well as cosmological observations of the sum of the neutrino masses for example with CMB-stage 4 [53] which aims to constrain the sum of the neutrino masses below $0.5 \mathrm{eV}$, and for experiments measuring the kinematic neutrino mass making our results possible benchmark points for these experiments.

Turning now to the mixing sum rules in the models considered. In figure 5 we show the correlations $\sin ^{2} \theta_{12}$ vs. $\sin ^{2} \theta_{13}$ and $\sin ^{2} \theta_{23}$ vs. $\delta$ respectively for both orderings. It should be noted that SR 1, case I and case II exhibit the same mixing sum rules such that we will refer to both cases simply as SR 1 . For all sum rules the predicted values of $\sin ^{2} \theta_{12}$ deviate from the currently preferred value. In the near future JUNO [54] will measure $\theta_{12}$ with a very good accuracy which will thoroughly probe these models. Due to the intimate relation between $\theta_{13}$ and $\theta_{12}$ here a precise measurement of one of these angles ultimately fixes the prediction for the other angle.

The situation for $\delta$ and $\theta_{23}$ is slightly different. Although also in this case we obtain strong correlations there are larger non-trivial regions allowed in the $\sin ^{2} \theta_{23}-\delta$ plane. Furthermore degeneracies are present such that a measurement of one parameter does not fix the other parameter uniquely in the considered cases. For example, for SR 1 in NO 

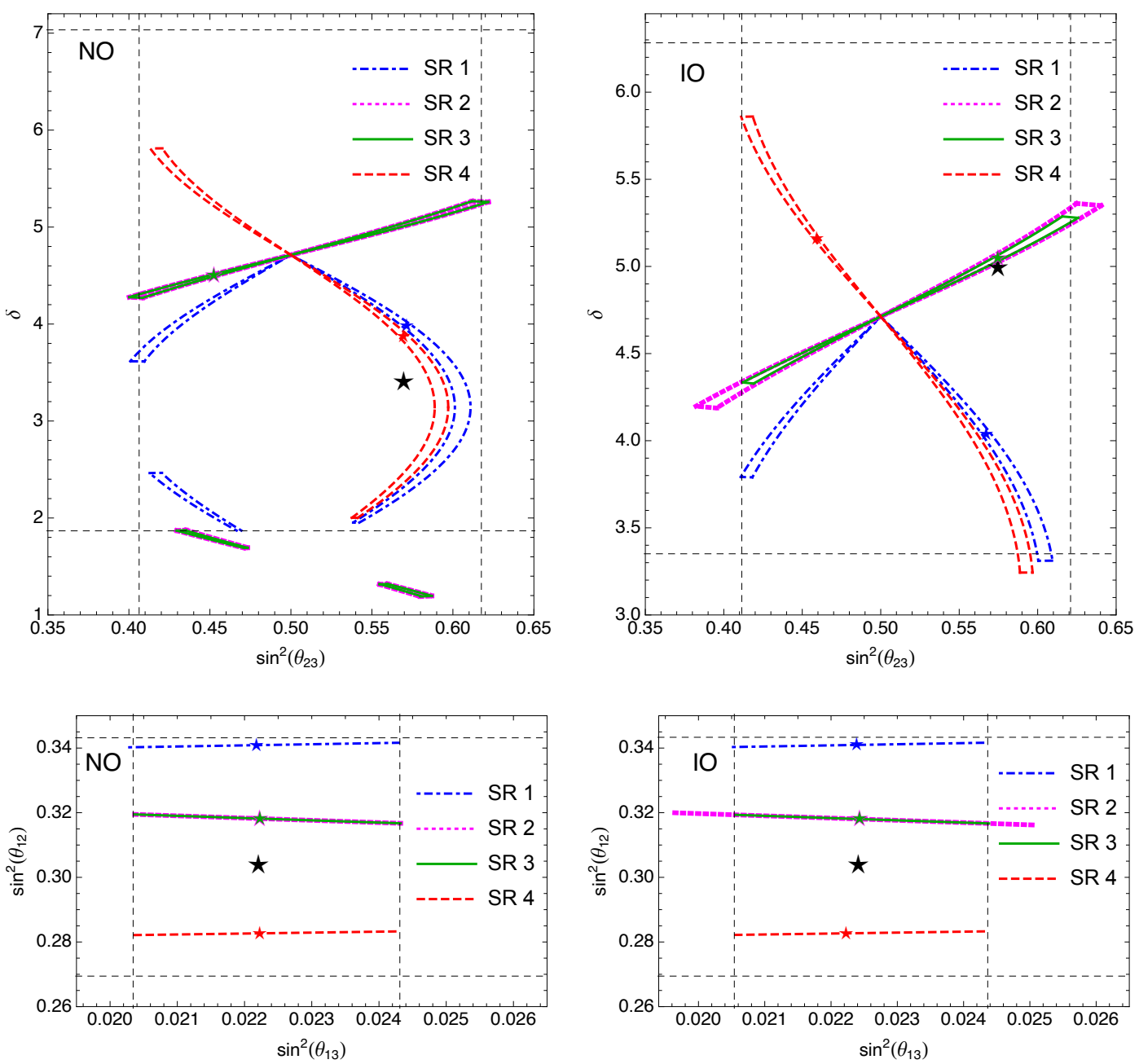

Figure 5. Allowed ranges for the mixing parameters for all sum rules using $\theta, \phi$ in their $3 \sigma$ ranges. The black dashed lines show the $3 \sigma$ allowed region without sum rules for NO/IO. The coloured (black) stars shows the best fits with (without) sum rules.

values of $\delta$ between 3.6 and 4.6 are allowed for two different values of $\sin ^{2} \theta_{23}$ in both octants due to a symmetry around $\sin ^{2} \theta_{23}=0.5$ in the expressions for $\delta$. In all cases the best fit value for $\delta$ is larger than $\pi$. And $\theta_{23}$ is preferred in the lower octant for SR 2 and 3 or in the upper octant for SR 1 and 4 in NO whereas in IO only SR 4 prefers $\theta_{23}$ in the lower octant. Maximal $\theta_{23}$ corresponds to $\delta$ equal or close to $3 \pi / 2$ in all models which can be easily understood analytically from the formulas in section 3. Furthermore, $\delta=\pi$ is only possible in NO for SR 1 and 2 for $\theta_{23}$ in the upper octant while in IO $\delta=\pi$ is excluded. Due to the input from the global fit, $\delta=0,2 \pi$ is not possible in any model independent of the mass ordering, and we find a preference for CPV in all models under consideration.

For easier comparison we also quote here for the Jarlskog invariant [55]

$$
J_{\mathrm{CP}}=\sin \theta_{13} \sin \theta_{12} \sin \theta_{23} \cos ^{2} \theta_{13} \cos \theta_{12} \cos \theta_{23} \sin \delta
$$


the $3 \sigma$ ranges (in units of $10^{-2}$ ) for the considered models

$$
\begin{array}{ll}
J_{\mathrm{CP}}^{\mathrm{NO}} \in[-3.60,3.40], & J_{\mathrm{CP}}^{\mathrm{IO}} \in[-3.61,-0.55] \text { for SR } 1, \\
J_{\mathrm{CP}}^{\mathrm{NO}} \in[-3.54,3.51], & J_{\mathrm{CP}}^{\mathrm{IO}} \in[-3.59,-2.47] \text { for SR } 2, \\
J_{\mathrm{CP}}^{\mathrm{NO}} \in[-3.54,3.50], & J_{\mathrm{CP}}^{\mathrm{IO}} \in[-3.54,-2.67] \text { for SR } 3, \\
J_{\mathrm{CP}}^{\mathrm{NO}} \in[-3.43,3.10], & J_{\mathrm{CP}}^{\mathrm{IO}} \in[-3.43,-0.32] \text { for SR } 4 .
\end{array}
$$

We now come to the non-trivial interplay between mass and mixing sum rules in the considered models. In figure 6 we show the dependence of $\sin ^{2} \theta_{23}$ or $\delta$ on the sum of the neutrino masses in the $3 \sigma$ ranges of the model parameters and the mass splittings. Interestingly, we observe similar dependencies and correlations in all cases.

SR 1 case I and II exhibit the same dependencies between $\sin ^{2} \theta_{23}$ or $\delta$ and $\sum m_{i}$ such that we refer to both cases as SR 1 in the following. This is easy to understand since in both cases the mixing sum rules are the same and the condition that the mass sum rule needs to be fulfilled, cf. eq. (2.4), only depends on the absolute values of $f_{1}$ and $f_{2}$ which are identical for both cases since they only differ in a phase. The situation is however different when deriving the predictions for $\left|m_{e e}\right|$ which explicitly depends on the phases of $f_{1}$ and $f_{2}$, see eq. (2.8).

For all sum rules a smaller mass scale leads to $\delta$ close to $\pi$ in NO such that the smallest mass in the model can only be achieved if $\delta \approx \pi$, whereas larger neutrino masses are only allowed for $\delta$ further away from $\pi$. However, we see the opposite effect in IO where a smaller mass scale allows for $\delta$ closer to $2 \pi$ for SR 2, 3 and 4 . Due to the interplay of the sum rules we find a clearly preferred region for the mass scale in the case of NO in particular for SR 1 and 4. This also explains why in [44] the authors find a preference for a mass scale. Nevertheless, in all four cases the cosmological upper bound on the mass scale can be saturated in the $3 \sigma$ range.

Also for the relation between $\sin ^{2} \theta_{23}$ and the sum of neutrino masses we note different effects depending on the mass ordering. For NO SR 2 and 3 present only a comparatively mild dependence of the allowed range of $\theta_{23}$ on the mass scale. For SR 1 and 4 on the other hand the dependence is stronger and we predict an upper and lower bound on the mass scale for $\sin ^{2} \theta_{23}>0.55$. For IO all sum rules present strong dependency of $\sin ^{2} \theta_{23}$ on the neutrino mass scale. For SR 2 and 3 smaller mass scales allow for larger values of $\theta_{23}$ such that $\theta_{23}$ can only lie in the lower octant if $\sum m_{i} \gtrsim 0.18 \mathrm{eV}$ whereas for SR 1 and 4 the majority of the parameter space predicts $\theta_{23}$ in the lower octant which also predicts a smaller mass scale. In fact, $\sin ^{2} \theta_{23}>0.5$ can only be achieved in these SR for $\sum m_{i} \gtrsim 0.2 \mathrm{eV}(\mathrm{SR} 4)$ or $\sum m_{i} \gtrsim 0.18 \mathrm{eV}$ (SR 1).

It should be noted that the predictions of these sum rules have been derived assuming only small renormalization group running effects. This is for example satisfied in the SM or in its supersymmetric extensions for small neutrino mass scales and small or moderate values of $\tan \beta$. However, as it has been shown, for instance, in [43] running effects can be large for mixing sum rules while conventional mass sum rules are in general largely unaffected by corrections [20, 21]. Since the sum rules in models based on modular symmetries 

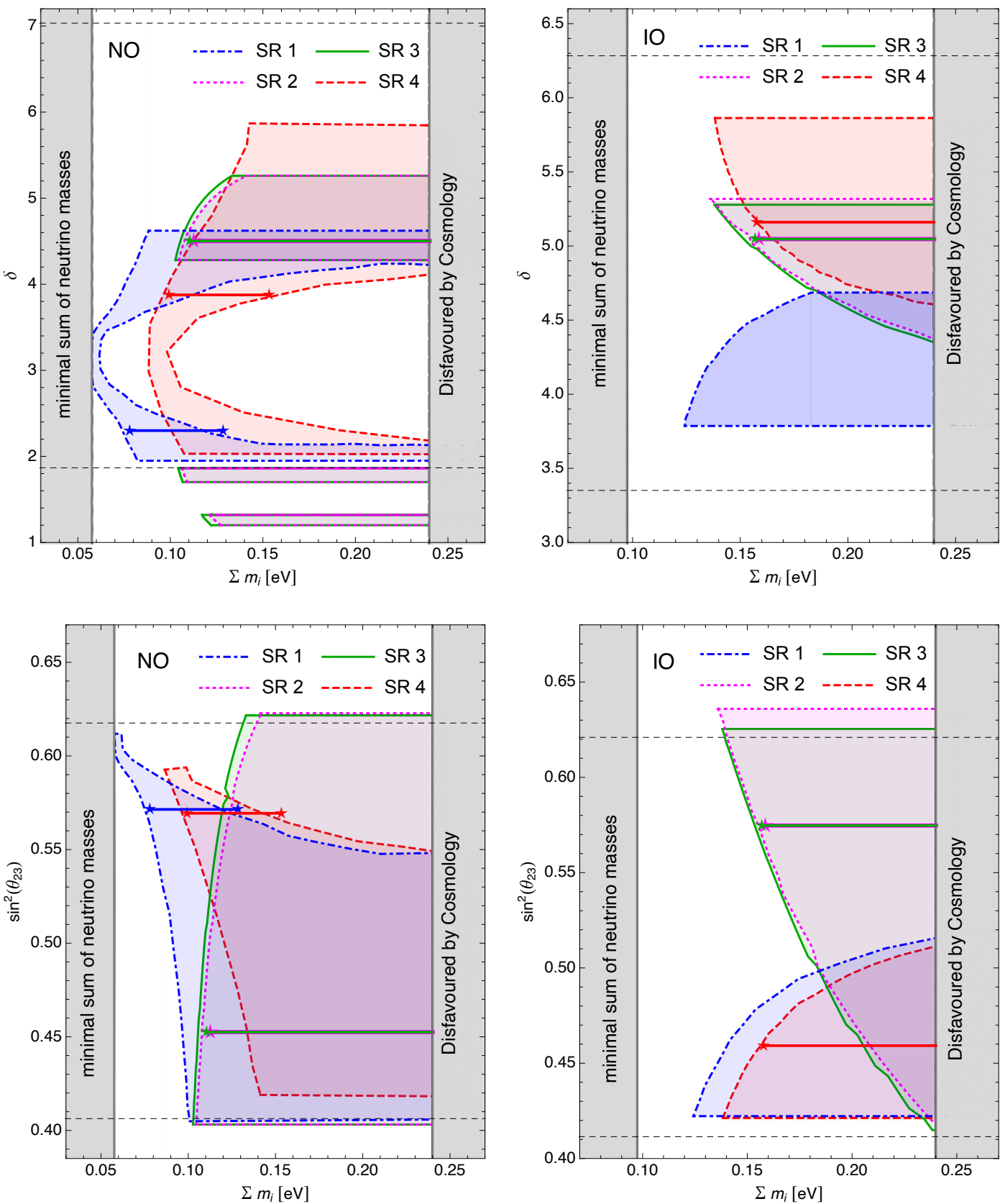

Figure 6. Allowed ranges for the mixing parameters depending on the sum of the neutrino masses for all SR in NO (left panels) and IO (right panels) for $\theta, \phi$ and the mass splittings in their $3 \sigma$ ranges. The stars and thick lines show the best fit regions in these models. Notice that for SR 1 , IO the mass sum rule cannot be fulfilled if $\theta, \phi$ are at best fit. The black dashed lines show the $3 \sigma$ allowed region without sum rules for $\mathrm{NO} / \mathrm{IO}$. The gray exclusion regions show the minimal value of the sum of the neutrino masses from oscillation experiments and the maximal value of the sum of the neutrino masses from cosmology. 
relate mass and mixing sum rules the previous statement needs to be reevaluated and we expect that running effects could be sizeable for mass sum rules in these cases as well.

\section{Summary and conclusions}

In this manuscript we have studied a new class of leptonic sum rules derived from flavour models based on modular symmetries. Specifically, we limited this study to models where a residual symmetry in the lepton sector is preserved. After deriving the analytical expressions for the sum rules we evaluated them numerically to show their predictions for upcoming neutrino experiments.

Due to the parameter reduction in these models we find relations between various observables. On the one hand there are mixing sum rules, which relate the mixing angles and the Dirac CPV phase and on the other hand there are mass sum rules, which connect the neutrino masses and Majorana phases. Similar relations have been already observed before in flavour models with conventional discrete symmetries. What is different here is that the coefficients of the mass sum rules are not constants anymore. They depend on the mixing parameters, which we parametrized in terms of only two parameters, an angle $\theta$ and a phase $\phi$. This feature leads to novel predictions, for example, we can get a preferred neutrino mass scale instead of just having a lower (and upper) bound.

This non-trivial interplay between mass and mixing sum rules leads to an interesting, distinct phenomenology compared to previous conventional models. Certain aspects have been observed before, like the close relation between $\theta_{12}$ and $\theta_{13}$ or the appearance of a lower bound of the neutrino mass scale. Other aspects are new. In particular, we would like to highlight here the correlation between the Dirac CPV phase and the atmospheric mixing angle to the allowed range for the neutrino mass scale. Some of our results have already been uncovered in purely numerical studies of these models but our analytical expressions provide better insights on the origin of these results. Hence, our work advances the study of models with modular symmetries from a theoretical point of view.

This paper demonstrates again the power of sum rules. It allowed us to write the predictions of four very different models in a unified framework such that we can directly compare them in terms of phenomenological predictions. That means sum rules are an ideal tool to provide benchmark scenarios for experimental studies. Due to their broad impact on various experiments ranging from oscillation experiments to neutrino experiments weighing the neutrino mass scale leptonic sum rules provide a variety of testable signatures. Our results here can also be immediately compared to previous systematic studies on mass sum rules, for instance, [16-21]. This work here shows how we can generalise that language to a completely new class of models with modular symmetries, which have recently been investigated in the literature.

Due to the insights provided in this manuscript, the improved comparability of results and the ease of application in experimental studies we hope that it will become a standard way to present predictions from flavour models in the future. 


\section{Acknowledgments}

We would like to thank Serguey Petcov for some very useful comments on this manuscript and insights into models with modular symmetry and Arsenii Titov for some help understanding their modular $A_{5}$ model. JG is supported by the US Department of Energy under Grant Contract DE-SC0012704. MS is supported by the Ministry of Science and Technology (MOST) of Taiwan under grant number MOST 107-2112-M-007-031-MY3.

Open Access. This article is distributed under the terms of the Creative Commons Attribution License (CC-BY 4.0), which permits any use, distribution and reproduction in any medium, provided the original author(s) and source are credited.

\section{References}

[1] I. Esteban, M.C. Gonzalez-Garcia, M. Maltoni, T. Schwetz and A. Zhou, The fate of hints: updated global analysis of three-flavor neutrino oscillations, JHEP 09 (2020) 178 [arXiv: 2007.14792] [INSPIRE].

[2] Particle Data Group collaboration, Review of Particle Physics, Phys. Rev. D 98 (2018) 030001 [INSPIRE].

[3] G. Altarelli and F. Feruglio, Discrete Flavor Symmetries and Models of Neutrino Mixing, Rev. Mod. Phys. 82 (2010) 2701 [arXiv: 1002.0211] [InSPIRE].

[4] H. Ishimori, T. Kobayashi, H. Ohki, Y. Shimizu, H. Okada and M. Tanimoto, Non-Abelian Discrete Symmetries in Particle Physics, Prog. Theor. Phys. Suppl. 183 (2010) 1 [arXiv: 1003.3552] [INSPIRE].

[5] S.F. King and C. Luhn, Neutrino Mass and Mixing with Discrete Symmetry, Rept. Prog. Phys. 76 (2013) 056201 [arXiv: 1301.1340] [INSPIRE].

[6] S.F. King, A. Merle, S. Morisi, Y. Shimizu and M. Tanimoto, Neutrino Mass and Mixing: from Theory to Experiment, New J. Phys. 16 (2014) 045018 [arXiv:1402.4271] [InSPIRE].

[7] F. Feruglio, Pieces of the Flavour Puzzle, Eur. Phys. J. C 75 (2015) 373 [arXiv: 1503.04071] [INSPIRE].

[8] S.T. Petcov, Discrete Flavour Symmetries, Neutrino Mixing and Leptonic CP-violation, Eur. Phys. J. C 78 (2018) 709 [arXiv:1711.10806] [INSPIRE].

[9] Z.-z. Xing, Flavor structures of charged fermions and massive neutrinos, Phys. Rept. 854 (2020) 1 [arXiv: 1909.09610] [inSPIRE].

[10] F. Feruglio and A. Romanino, Neutrino Flavour Symmetries, arXiv:1912.06028 [INSPIRE].

[11] G. Altarelli, F. Feruglio and C. Hagedorn, A SUSY SU(5) Grand Unified Model of Tri-Bimaximal Mixing from $A_{4}$, JHEP 03 (2008) 052 [arXiv:0802.0090] [INSPIRE].

[12] M. Hirsch, S. Morisi and J.W.F. Valle, Tri-bimaximal neutrino mixing and neutrinoless double beta decay, Phys. Rev. D 78 (2008) 093007 [arXiv:0804.1521] [INSPIRE].

[13] F. Bazzocchi, L. Merlo and S. Morisi, Phenomenological Consequences of See-Saw in $S_{4}$ Based Models, Phys. Rev. D 80 (2009) 053003 [arXiv: 0902 . 2849] [InSPIRE].

[14] G. Altarelli and D. Meloni, A Simplest A4 Model for Tri-Bimaximal Neutrino Mixing, J. Phys. G 36 (2009) 085005 [arXiv:0905.0620] [InSPIRE]. 
[15] M.-C. Chen and S.F. King, A4 See-Saw Models and Form Dominance, JHEP 06 (2009) 072 [arXiv:0903.0125] [INSPIRE].

[16] J. Barry and W. Rodejohann, Neutrino Mass Sum-rules in Flavor Symmetry Models, Nucl. Phys. B 842 (2011) 33 [arXiv:1007.5217] [INSPIRE].

[17] L. Dorame, D. Meloni, S. Morisi, E. Peinado and J.W.F. Valle, Constraining Neutrinoless Double Beta Decay, Nucl. Phys. B 861 (2012) 259 [arXiv:1111.5614] [InSPIRE].

[18] S.F. King, A. Merle and A.J. Stuart, The Power of Neutrino Mass Sum Rules for Neutrinoless Double Beta Decay Experiments, JHEP 12 (2013) 005 [arXiv:1307.2901] [INSPIRE].

[19] M. Agostini, A. Merle and K. Zuber, Probing flavor models with ${ }^{76}$ Ge-based experiments on neutrinoless double- $\beta$ decay, Eur. Phys. J. C 76 (2016) 176 [arXiv: 1506. 06133] [InSPIRE].

[20] J. Gehrlein, A. Merle and M. Spinrath, Renormalisation Group Corrections to Neutrino Mass Sum Rules, JHEP 09 (2015) 066 [arXiv:1506.06139] [InSPIRE].

[21] J. Gehrlein, A. Merle and M. Spinrath, Predictivity of Neutrino Mass Sum Rules, Phys. Rev. D 94 (2016) 093003 [arXiv: 1606. 04965] [INSPIRE].

[22] S.M. Bilenky, J. Hosek and S.T. Petcov, On Oscillations of Neutrinos with Dirac and Majorana Masses, Phys. Lett. B 94 (1980) 495 [INSPIRE].

[23] S.F. King, Predicting neutrino parameters from $\mathrm{SO}(3)$ family symmetry and quark-lepton unification, JHEP 08 (2005) 105 [hep-ph/0506297] [INSPIRE].

[24] I. Masina, A Maximal atmospheric mixing from a maximal CP-violating phase, Phys. Lett. B 633 (2006) 134 [hep-ph/0508031] [INSPIRE].

[25] A.D. Hanlon, S.-F. Ge and W.W. Repko, Phenomenological consequences of residual $\mathbb{Z}_{2}^{s}$ and $\overline{\mathbb{Z}}_{2}^{s}$ symmetries, Phys. Lett. B $\mathbf{7 2 9}$ (2014) 185 [arXiv:1308.6522] [INSPIRE].

[26] S.-F. Ge, D.A. Dicus and W.W. Repko, Residual Symmetries for Neutrino Mixing with a Large $\theta_{13}$ and Nearly Maximal $\delta_{D}$, Phys. Rev. Lett. 108 (2012) 041801 [arXiv:1108.0964] [INSPIRE].

[27] S.-F. Ge, D.A. Dicus and W.W. Repko, $\mathbb{Z}_{2}$ Symmetry Prediction for the Leptonic Dirac CP Phase, Phys. Lett. B 702 (2011) 220 [arXiv:1104.0602] [INSPIRE].

[28] D. Marzocca, S.T. Petcov, A. Romanino and M.C. Sevilla, Nonzero $\left|U_{e 3}\right|$ from Charged Lepton Corrections and the Atmospheric Neutrino Mixing Angle, JHEP 05 (2013) 073 [arXiv: 1302.0423] [INSPIRE].

[29] S.T. Petcov, Predicting the values of the leptonic CP-violation phases in theories with discrete flavour symmetries, Nucl. Phys. B 892 (2015) 400 [arXiv:1405.6006] [INSPIRE].

[30] I. Girardi, S.T. Petcov and A.V. Titov, Predictions for the Leptonic Dirac CP-violation Phase: a Systematic Phenomenological Analysis, Eur. Phys. J. C 75 (2015) 345 [arXiv: 1504.00658] [INSPIRE].

[31] I. Girardi, S.T. Petcov, A.J. Stuart and A.V. Titov, Leptonic Dirac CP-violation Predictions from Residual Discrete Symmetries, Nucl. Phys. B 902 (2016) 1 [arXiv:1509.02502] [INSPIRE].

[32] S.K. Agarwalla, S.S. Chatterjee, S.T. Petcov and A.V. Titov, Addressing Neutrino Mixing Models with DUNE and T2HK, Eur. Phys. J. C 78 (2018) 286 [arXiv:1711.02107] [INSPIRE]. 
[33] S.T. Petcov and A.V. Titov, Assessing the Viability of $A_{4}, S_{4}$ and $A_{5}$ Flavour Symmetries for Description of Neutrino Mixing, Phys. Rev. D 97 (2018) 115045 [arXiv:1804.00182] [INSPIRE].

[34] M. Blennow, M. Ghosh, T. Ohlsson and A. Titov, Testing Lepton Flavor Models at ESSnuSB, JHEP 07 (2020) 014 [arXiv:2004.00017] [INSPIRE].

[35] M. Blennow, M. Ghosh, T. Ohlsson and A. Titov, Probing Lepton Flavor Models at Future Neutrino Experiments, Phys. Rev. D 102 (2020) 115004 [arXiv:2005.12277] [INSPIRE].

[36] F. Feruglio, Are neutrino masses modular forms?, in From My Vast Repertoire ...: Guido Altarelli's Legacy, A. Levy, S. Forte and G. Ridolfi, eds., pp. 227-266, (2019), arXiv:1706.08749, DOI [INSPIRE].

[37] J. Gehrlein and M. Spinrath, Neutrino Mass Sum Rules and Symmetries of the Mass Matrix, Eur. Phys. J. C 77 (2017) 281 [arXiv:1704.02371] [InSPIRE].

[38] Planck collaboration, Planck 2018 results. VI. Cosmological parameters, Astron. Astrophys. 641 (2020) A6 [arXiv: 1807.06209] [INSPIRE].

[39] KATRIN collaboration, Improved Upper Limit on the Neutrino Mass from a Direct Kinematic Method by KATRIN, Phys. Rev. Lett. 123 (2019) 221802 [arXiv:1909.06048] [INSPIRE].

[40] P.P. Novichkov, S.T. Petcov and M. Tanimoto, Trimaximal Neutrino Mixing from Modular A4 Invariance with Residual Symmetries, Phys. Lett. B 793 (2019) 247 [arXiv:1812.11289] [INSPIRE].

[41] P.F. Harrison, D.H. Perkins and W.G. Scott, Tri-bimaximal mixing and the neutrino oscillation data, Phys. Lett. B 530 (2002) 167 [hep-ph/0202074] [INSPIRE].

[42] W. Grimus and L. Lavoura, A Model for trimaximal lepton mixing, JHEP 09 (2008) 106 [arXiv: 0809.0226] [INSPIRE].

[43] J. Gehrlein, S.T. Petcov, M. Spinrath and A.V. Titov, Renormalisation Group Corrections to Neutrino Mixing Sum Rules, JHEP 11 (2016) 146 [arXiv:1608.08409] [InSPIRE].

[44] P.P. Novichkov, J.T. Penedo and S.T. Petcov, Double cover of modular $S_{4}$ for flavour model building, Nucl. Phys. B 963 (2021) 115301 [arXiv:2006.03058] [INSPIRE].

[45] H. Okada and M. Tanimoto, Modular invariant flavor model of $A_{4}$ and hierarchical structures at nearby fixed points, Phys. Rev. D 103 (2021) 015005 [arXiv: 2009.14242] [INSPIRE].

[46] S.F. King and Y.-L. Zhou, Trimaximal TM1 mixing with two modular $S_{4}$ groups, Phys. Rev. D 101 (2020) 015001 [arXiv:1908.02770] [INSPIRE].

[47] I. de Medeiros Varzielas and L. Lavoura, Flavour models for TM $M_{1}$ lepton mixing, J. Phys. G 40 (2013) 085002 [arXiv:1212.3247] [INSPIRE].

[48] C. Luhn, Trimaximal TM $M_{1}$ neutrino mixing in $S_{4}$ with spontaneous CP-violation, Nucl. Phys. $B \mathbf{8 7 5}$ (2013) 80 [arXiv:1306.2358] [INSPIRE].

[49] P.P. Novichkov, J.T. Penedo, S.T. Petcov and A.V. Titov, Modular $S_{4}$ models of lepton masses and mixing, JHEP 04 (2019) 005 [arXiv: 1811.04933] [INSPIRE].

[50] P.P. Novichkov, J.T. Penedo, S.T. Petcov and A.V. Titov, Modular $A_{5}$ symmetry for flavour model building, JHEP 04 (2019) 174 [arXiv:1812.02158] [INSPIRE]. 
[51] L.L. Everett and A.J. Stuart, Icosahedral (A(5)) Family Symmetry and the Golden Ratio Prediction for Solar Neutrino Mixing, Phys. Rev. D 79 (2009) 085005 [arXiv:0812.1057] [INSPIRE].

[52] A.S. Barabash, Possibilities of future double beta decay experiments to investigate inverted and normal ordering region of neutrino mass, Front. in Phys. 6 (2019) 160 [arXiv: 1901.11342] [INSPIRE].

[53] CMB-S4 collaboration, CMB-S4 Science Book, First Edition, arXiv: 1610.02743 [INSPIRE].

[54] JUNO collaboration, Neutrino Physics with JUNO, J. Phys. G 43 (2016) 030401 [arXiv: 1507.05613] [INSPIRE].

[55] C. Jarlskog, Commutator of the Quark Mass Matrices in the Standard Electroweak Model and a Measure of Maximal CP-violation, Phys. Rev. Lett. 55 (1985) 1039 [INSPIRE]. 